\title{
Palladium(II)-Catalyzed Annulation of Alkynals with Ortho-Ester-Containing Phenylboronic Acids
}

\author{
Hirokazu Tsukamoto* and Yoshinori Kondo \\ Graduate School of Pharmaceutical Sciences, Tohoku University, \\ Aramaki-aza aoba 6-3, Aoba-ku, Sendai 980-8578, Japan
}

\section{Supplementary Material}

General Techniques. Reactions were carried out in clean, dry glassware under argon atmosphere using high quality solvents. Anhydrous solutions of reaction mixtures were transferred via an oven-dried cannula. Anhydrous methanol $(\mathrm{MeOH})$ was obtained by distillation from magnesium. Anhydrous $N, N$-dimethylformamide (DMF), acetonitrile, and 1,2-dichloroethane were obtained by distillation from calcium hydride. Anhydrous 1,4-dioxane and toluene were obtained by distillation from sodium benzophenone ketyl. Yields refer to chromatographically and spectroscopically homogenous materials. Reagents were purchased at the highest commercial quality and used without further purification. Reactions were monitored by thin layer chromatography separations carried out on $0.2 \mathrm{~mm}$ E. Merck silica gel plates (60 F254) using UV light as a visualizing agent and phosphomolybdic acid, $p$-anisaldehyde, or potassium permanganate solutions and heat as developing agents. Flash chromatographies were performed with Kanto silica gel 60 N (spherical, neutral, 63-210 $\mu \mathrm{m}$ grade). Purifications of $1 H$-inden-1-ols, $1 H$-inden-1-ones, and 2-naphthols were performed on $0.75 \mathrm{~mm}$ Wakogel ${ }^{\circledR}$ B-5F PLC plates. Further purification was performed on gel permeation chromatography (GPC) on a Japan Analytical Industry Model LC-918 (recycling preparative HPLC) using UV detector 3702 and refractive index detector RI-50. Melting points were obtained on a Yazawa apparatus with micro cover glass and are uncorrected. Infrared (IR) data were recorded on SensIR technologies' TravelIR ${ }^{\mathrm{TM}}$. Absorbance frequencies are reported in reciprocal centimeters $\left(\mathrm{cm}^{1}\right) .{ }^{1} \mathrm{H}$ and ${ }^{13} \mathrm{C}$ nuclear magnetic resonance (NMR) were recorded on JEOL AL 400 (400 and $100 \mathrm{MHz}$ respectively). Chemical shifts are reported in delta $(\delta)$ units, in parts per million ppm) relative to the singlet at $7.25 \mathrm{ppm}$ for chloroform- $d$. Splitting pattern are designated as $\mathrm{s}$, singlet; d, doublet; t, triplet; q, quartet; m, multiplet; and br, broad. Coupling constants $(J)$ are reported in Hertz $(\mathrm{Hz})$. Low and high resolution mass spectral (LRMS and HRMS) were obtained from Mass Spectrometry Resource, Graduate School of 
Pharmaceutical Sciences, Tohoku University, on a JEOL JMS-DX303 and JMS-700 spectrometer respectively.

\section{Preparation of Substrates.}

Arylboronic acids 2a, 2g, 12a, and $\mathbf{1 2 b}$ were purchased.

Arylboronic acid pinacol esters $\mathbf{2 b}$-e were prepared by Pd-catalyzed borylation ${ }^{1}$ of the corresponding aryl iodides ${ }^{2}$.

Methyl 2-(4,4,5,5-tetramethyl-1,3,2-dioxaborolan-2-yl)benzoate (2b) ${ }^{3}$

White solid. $\mathrm{R}_{f}=0.20$ (10\% EtOAc/hexane). Mp: $42-44{ }^{\circ} \mathrm{C}$. IR (neat): 2989, 1713, 1343 , 1304, 1272, 1256, 1131, 1108, 1061, 855, 758, $691 \mathrm{~cm}^{-1} .{ }^{1} \mathrm{H}$ NMR (400 M Hz, $\left.\mathrm{CDCl}_{3}\right)$ : ठ 7.94 (d, J=7.8 Hz, 1H), 7.53-7.48 (m, 2H), 7.44-7.37 (m, 1H), 3.91 (s, 3H), 1.42 (s, 12H). $\left.{ }^{13} \mathrm{C} \mathrm{NMR} \mathrm{(100} \mathrm{M} \mathrm{Hz,} \mathrm{CDCl}_{3}\right): \delta 168.2,133.2,132.0,131.6,128.8,128.6,84.0$, 52.3, 25.0. LRMS (EI) $\mathrm{m} / z$ (relative intensity) $262[\mathrm{M}]^{+}$(0.45), 261 (0.55), 204 (100), 189 (39), 163 (25), 149 (26), 131 (22). HRMS (EI, [M] $]^{+}$): calcd for $\mathrm{C}_{14} \mathrm{H}_{19} \mathrm{BO}_{4}$, 262.1376; found, 262.1373.

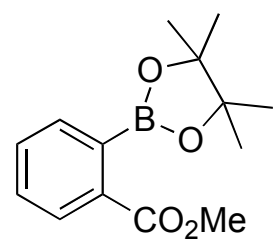

Ethyl 2-(4,4,5,5-tetramethyl-1,3,2-dioxaborolan-2-yl)benzoate (2c)

White solid. $\mathrm{R}_{f}=0.27$ (10\% EtOAc/hexane). Mp: $62-63^{\circ} \mathrm{C}$. IR (neat): 2981, 1704, 1341, 1301, 1281, 1140, 1061, $756 \mathrm{~cm}^{-1} .{ }^{1} \mathrm{H}$ NMR (400 M Hz, $\mathrm{CDCl}_{3}$ ): $\delta 7.93$ (d, J=7.8 Hz, 1H), 7.53-7.49 (m, 2H), 7.43-7.37 (m, 1H), 4.38 (q, J=7.2 Hz, 2H), $1.42(\mathrm{~s}, 12 \mathrm{H}), 1.38$ $(\mathrm{t}, J=7.2 \mathrm{~Hz}, 3 \mathrm{H}) .{ }^{13} \mathrm{C} \mathrm{NMR}\left(100 \mathrm{M} \mathrm{Hz}, \mathrm{CDCl}_{3}\right): \delta 167.8,133.7,131.9,131.5,128.7$, 128.4, 83.9, 61.2, 25.0, 14.5. LRMS (EI) $m / z$ (relative intensity) $276[\mathrm{M}]^{+}(0.44), 261$ (13), 218 (100), 189 (63), 149 (47), 131 (29). HRMS (EI, [M] ): calcd for $\mathrm{C}_{15} \mathrm{H}_{21} \mathrm{BO}_{4}$, 276.1533; found, 276.1514.<smiles>CCOc1ccccc1B1OC(C)(C)C(C)(C)O1</smiles>

iso-Propyl 2-(4,4,5,5-tetramethyl-1,3,2-dioxaborolan-2-yl)benzoate (2d) 
Colorless oil. $\mathrm{R}_{f}=0.32$ (10\% EtOAc/hexane). IR (neat): 2977, 1706, 1345, 1273, 1140, 1106, 1057, $749 \mathrm{~cm}^{-1} .{ }^{1} \mathrm{H}$ NMR (400 M Hz, $\mathrm{CDCl}_{3}$ ): $\delta 7.90$ (d, J=7.8 Hz, 1H), 7.49-7.48 (m, 2H), 7.41-7.35 (m, 1H), 5.29-5.20 (m, 1H), 1.42 (s, 12H), 1.35 (d, J=6.1 Hz, 6H). ${ }^{13} \mathrm{C}$ NMR (100 M Hz, $\left.\mathrm{CDCl}_{3}\right): \delta 167.3,134.2,131.8,131.4,128.5,128.1,83.9,68.7$, 25.0, 22.1. LRMS (EI) $m / z$ (relative intensity) $290[\mathrm{M}]^{+}$(0.18), 275 (3.9), 232 (58), 190 (100), 149 (33), 131 (17). HRMS (EI, [M-CH $]^{+}$): calcd for $\mathrm{C}_{15} \mathrm{H}_{20} \mathrm{BO}_{4}, 275.1455$; found, 275.1473 .<smiles>CCCOc1ccccc1B1OC(C)(C)C(C)(C)O1</smiles>

tert-Butyl 2-(4,4,5,5-tetramethyl-1,3,2-dioxaborolan-2-yl)benzoate (2e)

White solid. $\mathrm{R}_{f}=0.39$ (10\% EtOAc/hexane). Mp: $65-67^{\circ} \mathrm{C}$. IR (neat): 2975, 2931, 1702 , 1347, 1306, 1136, 1061, $753 \mathrm{~cm}^{-1} .{ }^{1} \mathrm{H}$ NMR (400 M Hz, $\left.\mathrm{CDCl}_{3}\right): \delta 7.81$ (d, J=7.8 Hz, $1 \mathrm{H}), 7.48-7.44(\mathrm{~m}, 2 \mathrm{H}), 7.39-7.33(\mathrm{~m}, 1 \mathrm{H}), 1.58(\mathrm{~s}, 9 \mathrm{H}), 1.41(\mathrm{~s}, 12 \mathrm{H}) .{ }^{13} \mathrm{C}$ NMR (100 $\left.\mathrm{M} \mathrm{Hz}, \mathrm{CDCl}_{3}\right): \delta 167.2,135.7,131.6,131.0,128.4,127.9,83.8,81.3,28.3,25.0$. LRMS

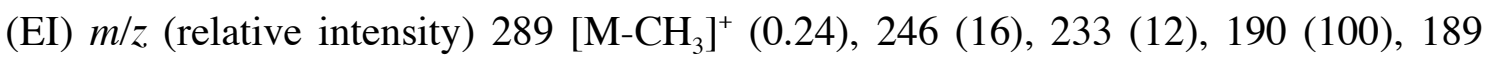
(58), 149 (32), 131 (15). HRMS (EI, [M] ): calcd for $\mathrm{C}_{17} \mathrm{H}_{25} \mathrm{BO}_{4}, 304.1846$; found, 304.1835 .<smiles>CCCCOc1ccccc1B1OC(C)(C)C(C)(C)O1</smiles>

$\mathrm{N}, \mathrm{N}$-Dimethyl-2-(4,4,5,5-tetramethyl-1,3,2-dioxaborolan-2-yl)benzamide (2f) was prepared by Pd-catalyzed borylation ${ }^{4}$ of 2-iodo- $N, N$-dimethylbenzamide ${ }^{5}$.

White solid. $\mathrm{R}_{f}=0.22$ (70\% EtOAc/hexane). Mp: $82-85^{\circ} \mathrm{C}$. IR (neat): 2970, 2923, 1630, 1156, 1028, 741, $695 \mathrm{~cm}^{-1} .{ }^{1} \mathrm{H}$ NMR (400 M Hz, $\mathrm{CDCl}_{3}$ ): $\delta 7.80$ (dd, J=7.3, $\left.1.4 \mathrm{~Hz}, 1 \mathrm{H}\right)$, 7.45 (ddd, $J=7.6,7.3,1.4 \mathrm{~Hz}, 1 \mathrm{H}$ ), 7.36 (ddd, $J=7.3,7.3,1.1 \mathrm{~Hz}, 1 \mathrm{H}$ ), 7.29 (dd, J=7.6, $1.1 \mathrm{~Hz}, 1 \mathrm{H}), 2.95$ (br-s, 6H), $1.31(\mathrm{~s}, 12 \mathrm{H}) .{ }^{13} \mathrm{C} \mathrm{NMR}\left(100 \mathrm{M} \mathrm{Hz}, \mathrm{CDCl}_{3}\right): \delta 172.2$, 142.4, 134.8, 130.7, 128.0, 125.4, 83.5, 25.1. LRMS (EI) $\mathrm{m} / \mathrm{z}$ (relative intensity) 275 $[\mathrm{M}]^{+}$(9.0), 274 (21), 260 (10), 217 (100), 216 (57), 144 (29). HRMS (EI, [M] ): calcd for $\mathrm{C}_{15} \mathrm{H}_{22} \mathrm{BNO}_{3}, 275.1693$; found, 275.1666. 


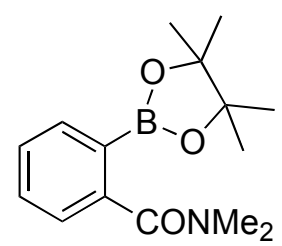

Arylboronic acid pinacol esters 14a-c were prepared by Pd-catalyzed borylation ${ }^{1}$ of methyl (2-iodophenyl)acetate ${ }^{6}$, dimethyl 2-(2-iodophenyl)malonate ${ }^{7}$, and methyl (2-iodophenyl)[(4-methylphenyl)sulfonyl]acetate. The latter two iodides were prepared by treatment of methyl (2-iodophenyl)acetate ${ }^{6}$ and 1-iodo-2-[[(4-methylphenyl)sulfonyl]methyl]benzene ${ }^{8}$ with dimethyl cabonate according to the literature procedure ${ }^{9}$.

Methyl [2-(4,4,5,5-tetramethyl-1,3,2-dioxaborolan-2-yl)phenyl]acetate (14a)

White solid. $\mathrm{R}_{f}=0.43$ (10\% EtOAc/toluene). Mp: $66-68^{\circ} \mathrm{C}$. IR (neat): $2975,1740,1343$, 1316, 1212, 1162, 1144, 1071, 768, $695 \mathrm{~cm}^{-1} .{ }^{1} \mathrm{H}$ NMR (400 M Hz, $\left.\mathrm{CDCl}_{3}\right): \delta 7.83$ (d, $J=7.2 \mathrm{~Hz}, 1 \mathrm{H}), 7.38$ (dd, J=7.4, 7.6 Hz, 1H), 7.27 (dd, J=7.2, $7.4 \mathrm{~Hz}, 1 \mathrm{H}), 7.19$ (d, $J=7.6 \mathrm{~Hz}, 1 \mathrm{H}), 3.98(\mathrm{~s}, 2 \mathrm{H}), 3.66(\mathrm{~s}, 3 \mathrm{H}), 1.32$ (s, 12H). ${ }^{13} \mathrm{C}$ NMR (100 M Hz, $\left.\mathrm{CDCl}_{3}\right): \delta 172.6,140.2,135.9,130.8,129.9,126.2$, 83.6, 51.8, 41.0, 25.0. LRMS (EI) $\mathrm{m} / z$ (relative intensity) $276[\mathrm{M}]^{+}$(0.63), 261 (6.8), 245 (9.2), 218 (100), 203 (31), 176 (20), 175 (18), 131 (19), 117 (23). HRMS (EI, [M] $]^{+}$): calcd for $\mathrm{C}_{15} \mathrm{H}_{21} \mathrm{BO}_{4}, 276.1533$; found, 276.1538.

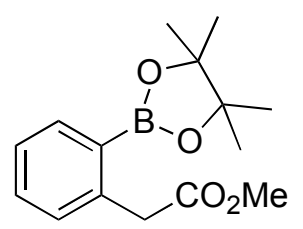

Dimethyl 2-(2-iodophenyl)malonate

White solid. $\mathrm{R}_{f}=0.48$ (10\% EtOAc/toluene). Mp: $62-64{ }^{\circ} \mathrm{C}$. IR (neat): $2946,1750,1735$, 1432, 1262, 1214, 1148, 1009, 990, $747 \mathrm{~cm}^{-1} .{ }^{1} \mathrm{H}$ NMR (400 M Hz, $\left.\mathrm{CDCl}_{3}\right): \delta 7.87$ (d, $J=7.8 \mathrm{~Hz}, 1 \mathrm{H}), 7.47$ (dd, $J=7.8,1.5 \mathrm{~Hz}, 1 \mathrm{H}), 7.38$ (dd, $J=7.8,7.7 \mathrm{~Hz}, 1 \mathrm{H}), 7.02$ (ddd, $J=7.8,7.7,1.5 \mathrm{~Hz}, 1 \mathrm{H}), 5.18(\mathrm{~s}, 1 \mathrm{H}), 3.78(\mathrm{~s}, 6 \mathrm{H}) .{ }^{13} \mathrm{C} \mathrm{NMR}\left(100 \mathrm{M} \mathrm{Hz}, \mathrm{CDCl}_{3}\right): \delta$ 168.1, 139.6, 136.1, 129.9, 129.7, 128.6, 101.5, 61.9, 53.0. LRMS (EI) $\mathrm{m} / \mathrm{z}$ (relative intensity) $334[\mathrm{M}]^{+}$(15), 207 (100), 147 (44), 133 (22). HRMS (EI, [M] $]^{+}$): calcd for $\mathrm{C}_{11} \mathrm{H}_{11} \mathrm{IO}_{4}, 333.9702$; found, 333.9687.<smiles>COC(=O)C(C(C)=O)c1ccccc1I</smiles> 
Dimethyl 2-[2-(4,4,5,5-tetramethyl-1,3,2-dioxaborolan-2-yl)phenyl]malonate (14b) Colorless oil. $\mathrm{R}_{f}=0.41$ (10\% EtOAc/toluene). IR (neat): 2977, 1754, 1735, 1436, 1347, 1314, 1144, 1119, 857, $762 \mathrm{~cm}^{-1} .{ }^{1} \mathrm{H}$ NMR (400 M Hz, $\mathrm{CDCl}_{3}$ ): $\delta 7.86$ (dd, J=7.2, 1.0 Hz, 1H), 7.45 (ddd, J=7.4, 7.2, $1.0 \mathrm{~Hz}, 1 \mathrm{H}), 7.39$ (dd, J=7.4, $1.2 \mathrm{~Hz}, 1 \mathrm{H}), 7.32$ (ddd, $J=7.2,7.2,1.2 \mathrm{~Hz}, 1 \mathrm{H}), 5.77$ (s, 1H), 3.75 (s, 6H), 1.33 (s, 12H). ${ }^{13} \mathrm{C}$ NMR $(100 \mathrm{M} \mathrm{Hz}$, $\left.\mathrm{CDCl}_{3}\right): \delta 169.6,138.9,136.3,131.3,128.4,127.2,83.9,55.9,52.5,24.8$. LRMS (EI) $m / z$ (relative intensity) $334[\mathrm{M}]^{+}(0.20), 319$ (8.1), 276 (100), 217 (45), 203 (20), 190 (48), 147 (11), 133 (9.9). HRMS (EI, [M] ${ }^{+}$): calcd for $\mathrm{C}_{17} \mathrm{H}_{23} \mathrm{BO}_{6}, 334.1588$; found, 334.1606.

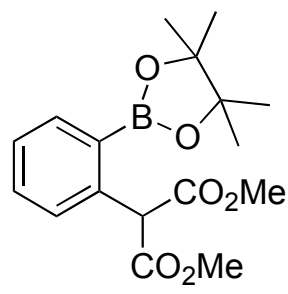

Methyl (2-iodophenyl)[(4-methylphenyl)sulfonyl]acetate

White solid. $\mathrm{R}_{f}=0.49$ (10\% EtOAc/toluene). Mp: $96-99{ }^{\circ} \mathrm{C}$. IR (neat): $2952,1746,1329$, 1270, 1140, 1082, 911, 801, $708 \mathrm{~cm}^{-1} .{ }^{1} \mathrm{H} \mathrm{NMR}\left(400 \mathrm{M} \mathrm{Hz}, \mathrm{CDCl}_{3}\right): \delta 7.98$ (d, J=7.9 Hz, 1H), 7.78 (d, J=7.9 Hz, 1H), 7.56 (d, $J=8.6 \mathrm{~Hz}, 2 \mathrm{H}), 7.39$ (dd, $J=7.9,7.6 \mathrm{~Hz}, 1 \mathrm{H}), 7.25$ (d, J=8.6 Hz, 2H), 7.05 (dd, J=7.9, 7.6 Hz, 1H), 5.77 (s, 1H), 3.00 (s, 3H), 2.43 (s, 3H). ${ }^{13} \mathrm{C}$ NMR $\left(100 \mathrm{M} \mathrm{Hz}, \mathrm{CDCl}_{3}\right): \delta 164.5,145.3,139.5,134.2,131.1,130.95,130.90$, $129.4 \times 2,128.3,103.5,77.2,53.4,21.8$. LRMS (EI) $\mathrm{m} / z$ (relative intensity) $430[\mathrm{M}]^{+}$ (14), 303 (17), 275 (68), 244 (18), 148 (100), 133 (17). HRMS (EI, [M] ): calcd for $\mathrm{C}_{16} \mathrm{H}_{15} \mathrm{IO}_{4} \mathrm{~S}, 429.9736$; found, 429.9717.<smiles>CC(=O)C([18O])c1ccccc1I</smiles>

Methyl

[2-(4,4,5,5-tetramethyl-1,3,2-dioxaborolan-2-yl)phenyl][(4-methylphenyl)sulfonyl]aceta te $(\mathbf{1 4 c})$

White amorphous mass. $\mathrm{R}_{f}=0.44$ (10\% EtOAc/toluene). IR (neat): 2979, 1744, 1345, 1318, 1287, 1142, 1117, 1082, 857, $758 \mathrm{~cm}^{-1} .{ }^{1} \mathrm{H}$ NMR (400 M Hz, $\mathrm{CDCl}_{3}$ ): $\delta 7.83$ (dd, $J=7.1,1.7 \mathrm{~Hz}, 1 \mathrm{H}), 7.69$ (d, $J=7.6 \mathrm{~Hz}, 1 \mathrm{H}), 7.47$ (d, $J=8.1 \mathrm{~Hz}, 2 \mathrm{H}), 7.40$ (ddd, $J=7.6$, 7.3, 1.7 Hz, 1H), 7.36 (dd, J=7.1, $7.3 \mathrm{~Hz}, 1 \mathrm{H}), 7.18$ (d, J=8.1 Hz, 2H), 6.63 (s, 1H), 3.74 (s, 3H), 2.39 (s, 3H), 1.35 (s, 6H), 1.29 (s, 6H). ${ }^{11} \mathrm{~B}$ NMR (128 M Hz, $\mathrm{CDCl}_{3}$ ): $\delta 30.1$. 
${ }^{13} \mathrm{C}$ NMR $\left(100 \mathrm{M} \mathrm{Hz}, \mathrm{CDCl}_{3}\right): \delta 165.9,144.5,136.2,134.3,133.6,130.7,129.7,129.5$, 128.8, 128.4, 84.1, 71.2, 53.0, 25.1, 24.8, 21.7. LRMS (EI) $\mathrm{m} / \mathrm{z}$ (relative intensity) 430 $\left[\mathrm{M}^{+}\right.$(2.9), 399 (3.9), 214 (19), 275 (100), 217 (37), 147 (69), 133 (44). HRMS (EI, $[\mathrm{M}]^{+}$): calcd for $\mathrm{C}_{22} \mathrm{H}_{27} \mathrm{BO}_{6} \mathrm{~S}, 430.1621$; found, 430.1626 .

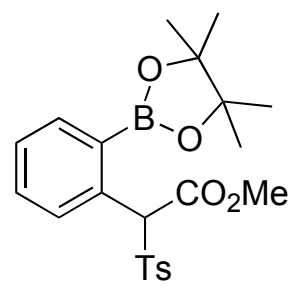

Internal alkynes $\mathbf{3 a}, \mathbf{3 d}, \mathbf{3 f}$, and $\mathbf{3 g}$ were purchased.

Diarylacetylenes $\mathbf{3 b}$ and $\mathbf{3 c}$ were prepared by Sonogashira reaction ${ }^{10}$ of 4 -iodoarene with 4-ethynylarene. The ${ }^{1} \mathrm{H},{ }^{13} \mathrm{C}$ NMR, and LRMS spectra were agreed with the reported value. ${ }^{11}$

Cyclododecyne $\mathbf{3 e}$ was prepared according to the literature procedure. ${ }^{12}$

\section{Preparation of Catalysts.}

Palladium catalysts $\mathbf{1 0 a - d}$ were prepared according to the literature procedures. ${ }^{13}$

\section{$\operatorname{Pd}(\mathrm{OAc})_{2}(\mathrm{dppe})-$ Catalyzed Annulation of 3d with 12a (Scheme 3):}

To a test tube containing 3d (11.3 mg, $0.103 \mathrm{mmol})$, 12a (18.4 mg, $0.123 \mathrm{mmol})$, and $\mathrm{Pd}(\mathrm{OAc})_{2}(\mathrm{dppe})(3.5 \mathrm{mg}, 5.6 \mu \mathrm{mol})$ was added anhydrous $\mathrm{MeOH}(0.5 \mathrm{~mL})$ under argon. The resulting mixture was sealed with a screw cap and agitated at room temperature for $24 \mathrm{~h}$. Then, $N, N$-diethanolaminomethyl polystyrene ${ }^{14}$ (PS-DEAM $^{\mathrm{TM}}, 1.63 \mathrm{mmol} / \mathrm{g}, 0.15$ $\mathrm{g}, 0.24 \mathrm{mmol})$ and THF $(2 \mathrm{~mL})$ were added to remove an excess of 12a. The mixture was agitated at room temperature for $2 \mathrm{~h}$. The mixture was filtered and thoroughly washed with $\mathrm{CHCl}_{3}$. The filtrate was concentrated in vacuo and the residue was purified by preparative TLC eluting with $1 \%$ EtOAc/toluene repeated three times to yield 2,3-dipropyl-1H-inden-1-ol ${ }^{15}$ (13a) (19.7 mg, $\left.0.0911 \mathrm{mmol}, 89 \%\right)$.

Pale yellow solid. $\mathrm{R}_{f}=0.46$ (30\% EtOAc/hexane). Mp: 49-54 ${ }^{\circ} \mathrm{C}$. IR (neat): 3128, 2956, 2927, 2867, 1463, 1198, 1096, 1061, 1038, 1003, 764, $727 \mathrm{~cm}^{-1} .{ }^{1} \mathrm{H}$ NMR (400 M Hz, $\left.\mathrm{CDCl}_{3}\right): \delta 7.46(\mathrm{~d}, J=7.0 \mathrm{~Hz}, 1 \mathrm{H}), 7.26(\mathrm{dd}, J=7.0,7.8 \mathrm{~Hz}, 1 \mathrm{H}), 7.15(\mathrm{~d}, J=7.8 \mathrm{~Hz}, 1 \mathrm{H})$, 7.14 (dd, $J=7.8,7.8 \mathrm{~Hz}, 1 \mathrm{H}), 4.97$ (s, 1H), 2.44-2.37 (m, 4H), 1.69-1.47 (m, 4H), 1.43 (br-s, 1H), $0.98(\mathrm{t}, J=7.3 \mathrm{~Hz}, 1 \mathrm{H}), 0.97(\mathrm{t}, J=7.3 \mathrm{~Hz}, 1 \mathrm{H}) .{ }^{13} \mathrm{C}$ NMR (100 M Hz, $\left.\mathrm{CDCl}_{3}\right): \delta 144.8,144.6,143.9,137.5,127.9,124.6,122.7,118.4,76.9,27.7,27.2,22.8$, 
21.7, 14.3x2. LRMS (EI) $m / z$ (relative intensity) $216[\mathrm{M}]^{+}$(60), 187 (100), 173 (62), 145 (71). HRMS (EI, [M] $]^{+}$): calcd for $\mathrm{C}_{15} \mathrm{H}_{20} \mathrm{O}, 216.1514$; found, 216.1501 .<smiles>CCCC1=C(P)C(O)c2ccccc21</smiles>

\section{$\operatorname{Pd}(\mathrm{OAc})_{2}$ (dppe)-Catalyzed Annulation of 3d with 12b (Scheme 3):}

To a test tube containing 3d (11.2 mg, $0.102 \mathrm{mmol})$, 12b $(21.1 \mathrm{mg}, 0.129 \mathrm{mmol})$, and $\mathrm{Pd}(\mathrm{OAc})_{2}($ dppe $)(3.3 \mathrm{mg}, 5.3 \mu \mathrm{mol})$ was added anhydrous $\mathrm{MeOH}(0.5 \mathrm{~mL})$ under argon. The resulting mixture was sealed with a screw cap and agitated at $50{ }^{\circ} \mathrm{C}$ for $24 \mathrm{~h}$. The mixture was cooled down to room temperature, and then PS-DEAM ${ }^{\mathrm{TM}}(1.63 \mathrm{mmol} / \mathrm{g}$, $0.15 \mathrm{~g}, 0.24 \mathrm{mmol})$ and THF (2 $\mathrm{mL})$ were added to remove an excess of $\mathbf{1 2 b}$. The mixture was agitated at room temperature for $2 \mathrm{~h}$. The mixture was filtered and thoroughly washed with $\mathrm{CHCl}_{3}$. The filtrate was concentrated in vacuo and the residue was purified by preparative TLC eluting with $1 \%$ EtOAc/toluene repeated three times to yield 1-methyl-2,3-dipropyl-1H-inden-1-ol ${ }^{15}$ (13b) (22.1 mg, 0.0959 mmol, 94\%).

White solid. $\mathrm{R}_{f}=0.51$ (30\% EtOAc/hexane). Mp: $74-80^{\circ} \mathrm{C}$. IR (neat): 3299, 2956, 2931, 2869, 1466, 1455, 1395, 1378, 1092, 1079, 938, $753 \mathrm{~cm}^{-1} .{ }^{1} \mathrm{H}$ NMR (400 M Hz, $\left.\mathrm{CDCl}_{3}\right)$ :

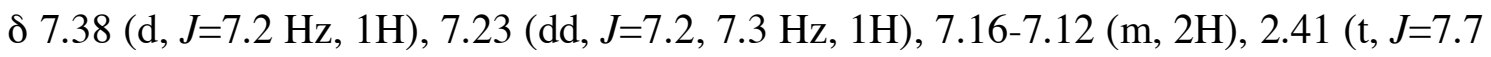
$\mathrm{Hz}, 2 \mathrm{H}), 2.37-2.24$ (m, 2H), 1.67-1.54 (m, 4H), 1.51 (s, 3H), 1.45 (br-s, 1H), 1.00 (t, $J=7.1 \mathrm{~Hz}, 1 \mathrm{H}), 0.98(\mathrm{t}, J=7.3 \mathrm{~Hz}, 1 \mathrm{H}) .{ }^{13} \mathrm{C} \mathrm{NMR}\left(100 \mathrm{M} \mathrm{Hz}, \mathrm{CDCl}_{3}\right): \delta 149.4,147.8$, $142.9,136.5,128.1,125.1,121.2,118.8,82.6,27.5,27.3,23.7,23.1,21.8,14.9,14.4$. LRMS (EI) $m / z$ (relative intensity) $230[\mathrm{M}]^{+}$(58), 201 (19), 187 (100), 159 (22), 145 (21). HRMS (EI, [M] $]^{+}$): calcd for $\mathrm{C}_{16} \mathrm{H}_{22} \mathrm{O}, 230.1671$; found, 230.1657.

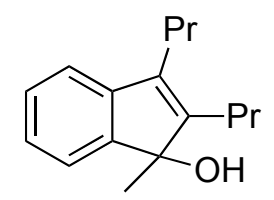

\section{Optimizations for Pd(II)-Catalyzed Synthesis of Indenone 7a (Table 1):}

To a test tube containing 3a (18 mg, $0.10 \mathrm{mmol}$, see Table $1 \mathrm{~S})$, aryl-boronic acids or -boronates $2(0.12 \mathrm{mmol})$, and $\mathrm{Pd}(\mathrm{II})$ catalyst $(5 \mu \mathrm{mol})$ was added anhydrous solvent (MeOH, DMF, acetonitrile, THF, 1,4-dioxane, 1,2-dichloroethane, or toluene, $0.5 \mathrm{~mL}$ ) under argon. The resulting mixture was sealed with a screw cap and agitated at $80{ }^{\circ} \mathrm{C}$ for the time described in Table 1. The mixture was cooled down to room temperature, 
and then PS-DEAM ${ }^{\mathrm{TM}}(1.63 \mathrm{mmol} / \mathrm{g}, 0.15 \mathrm{~g}, 0.24 \mathrm{mmol})$ and THF $(2 \mathrm{~mL})$ were added to remove an excess of 2 . The mixture was agitated at room temperature for $2 \mathrm{~h}$. The mixture was filtered and thoroughly washed with $\mathrm{CHCl}_{3}$. The filtrate was concentrated in vacuo and the residue was purified by preparative TLC eluting with $1 \%$ EtOAc/toluene repeated two times to yield 2,3-diphenyl- $1 H$-inden-1-one ${ }^{16}$ (7a).

Red solid. $\mathrm{R}_{f}=0.53$ (30\% EtOAc/hexane). Mp: $152-156^{\circ} \mathrm{C}$. IR (neat): $3070,1702,1347$, 1081, 780, 760, 751, 724, 699, $675 \mathrm{~cm}^{-1} .{ }^{1} \mathrm{H}$ NMR (400 M Hz, $\left.\mathrm{CDCl}_{3}\right): \delta 7.59$ (d, J=6.8 $\mathrm{Hz}, 1 \mathrm{H}), 7.40-7.25(\mathrm{~m}, 12 \mathrm{H}), 7.15(\mathrm{~d}, J=7.1 \mathrm{~Hz}, 1 \mathrm{H}) .{ }^{13} \mathrm{C} \mathrm{NMR}\left(100 \mathrm{M} \mathrm{Hz}, \mathrm{CDCl}_{3}\right): \delta$ 196.2, 155.1, 145.0, 133.3, 132.6, 132.2, 130.60, 130.57, 129.8, 129.1, 128.8, 128.6, 128.4, 127.9, 127.6, 122.8, 121.1. LRMS (EI) $\mathrm{m} / \mathrm{z}$ (relative intensity) $282[\mathrm{M}]^{+}(100)$, 252 (41), 126 (13). HRMS (EI, [M] $]^{+}$): calcd for $\mathrm{C}_{21} \mathrm{H}_{14} \mathrm{O}, 282.1045$; found, 282.1041.<smiles>O=C1C(=C(c2ccccc2)c2ccccc2)c2ccccc21</smiles> 
Table 1S

\begin{tabular}{|c|c|c|c|c|}
\hline entry & $\begin{array}{c}\mathbf{3 a} \\
(\mathrm{mg}, \mathrm{mmol})\end{array}$ & $\begin{array}{c}\mathbf{2} \\
(\mathrm{mg}, \mathrm{mmol})\end{array}$ & $\begin{array}{c}{[\mathrm{Pd}]} \\
(\mathrm{mg}, \mu \mathrm{mol})\end{array}$ & $\begin{array}{c}\mathbf{5 a} \\
(\mathrm{mg}, \mathrm{mmol}, \%)\end{array}$ \\
\hline 1 & $17.7,0.0993$ & $\mathbf{2 a} ; 23.2,0.129$ & 6a; $3.1,5.0$ & $26.0,0.0921,93$ \\
\hline 2 & $18.2,0.102$ & $\mathbf{2 a} ; 22.8,0.127$ & $\mathbf{6 a} ; 3.4,5.5$ & $16.5,0.0584,57$ \\
\hline 3 & $17.7,0.0993$ & $\mathbf{2 a} ; 23.1,0.128$ & $\mathbf{6 a} ; 3.4,5.5$ & $8.6,0.0305,31$ \\
\hline 4 & $17.8,0.0999$ & $\mathbf{2 a} ; 22.9,0.127$ & 6a; $3.1,5.0$ & $6.9,0.0244,24$ \\
\hline 5 & $17.7,0.0993$ & $\mathbf{2 a} ; 22.7,0.126$ & $\mathbf{6 a} ; 3.3,5.3$ & $3.5,0.0124,12$ \\
\hline 6 & $18.4,0.103$ & $\mathbf{2 a} ; 22.0,0.122$ & 6a; $3.1,5.0$ & $2.3,0.0081,8$ \\
\hline 7 & $17.8,0.0999$ & $2 \mathbf{a} ; 22.5,0.125$ & $6 \mathbf{6 a} ; 3.2,5.1$ & $0.3,0.0011,1$ \\
\hline 8 & $18.3,0.103$ & $\mathbf{2 a} ; 22.0,0.122$ & $\begin{array}{c}\mathrm{Pd}(\mathrm{OAc})_{2} ; 1.4,6.2 \\
\text { dppe; } 2.4,6.0\end{array}$ & $25.2,0.0893,87$ \\
\hline 9 & $18.2,0.102$ & $\mathbf{2 a} ; 22.2,0.123$ & $\mathrm{Pd}(\mathrm{OAc})_{2} ; 1.2,5.3$ & $0,0,0$ \\
\hline 10 & $18.6,0.104$ & $\mathbf{2 a} ; 23.2,0.129$ & $\begin{array}{c}\mathrm{Pd}(\mathrm{OAc})_{2}\left(\mathrm{PPh}_{2}\right)_{2} \\
4.0,5.3\end{array}$ & $0,0,0$ \\
\hline 11 & $17.9,0.100$ & $\mathbf{2 a} ; 22.4,0.124$ & $\mathrm{Pd}(\mathrm{dppe})_{2} ; 4.5,5.0$ & $6.4,0.0227,23$ \\
\hline 12 & $17.8,0.0999$ & $\mathbf{2 a} ; 22.8,0.127$ & $\mathbf{6 b} ; 3.1,5.4$ & $2.7,0.0096,10$ \\
\hline 13 & $18.1,0.102$ & $\mathbf{2 a} ; 23.0,0.128$ & $\mathbf{6 c} ; 4.1,5.6$ & $27.2,0.0963,95$ \\
\hline 14 & $17.6,0.0987$ & $\mathbf{2 a} ; 22.1,0.123$ & $\mathbf{6 d} ; 5.9,5.0$ & $18.8,0.0666,67$ \\
\hline 15 & $17.6,0.0987$ & $\mathbf{2 b} ; 31.3,0.128$ & $\mathbf{6 c} ; 3.9,5.3$ & $29.5,0.104,100$ \\
\hline 16 & $17.7,0.0993$ & $\mathbf{2 c} ; 33.6,0.122$ & $\mathbf{6 c} ; 3.8,5.2$ & $27.7,0.0981,99$ \\
\hline 17 & $17.8,0.0999$ & $\mathbf{2 d} ; 35.8,0.123$ & $\mathbf{6 c} ; 3.8,5.2$ & $25.8,0.0914,91$ \\
\hline 18 & $17.6,0.0987$ & $\mathbf{2 e} ; 38.5,0.127$ & $\mathbf{6 c} ; 3.9,5.3$ & $5.5,0.0195,20$ \\
\hline 19 & $18.4,0.103$ & 2f; $33.6,0.122$ & $\mathbf{6 c} ; 4.0,5.5$ & $4.5,0.0159,15$ \\
\hline 20 & $18.0,0.101$ & $2 \mathrm{~g} ; 19.0,0.129$ & $\mathbf{6 c} ; 4.0,5.5$ & $5.6,0.0198,20$ \\
\hline
\end{tabular}

\section{Pd(II)-Catalyzed Synthesis of Indenones 7 (Table 2):}

To a test tube containing 3a (1 equiv, see Table $2 \mathrm{~S}$ ), 2a (1.2 equiv), and $\operatorname{Pd}(\mathrm{II})$ catalyst (5 mol\%) was added anhydrous $\mathrm{MeOH}$ for entries 1, 3, 4, 5, and 6 or anhydrous DMF-MeOH (1:1) for entry $2(0.2 \mathrm{M})$ under argon. The resulting mixture was sealed with a screw cap and agitated at $80{ }^{\circ} \mathrm{C}$ for $2 \mathrm{~h}$. The mixture was cooled down to room temperature, and then PS-DEAM ${ }^{\mathrm{TM}}(1.63 \mathrm{mmol} / \mathrm{g}, 2.4$ equiv, $\mathrm{X} \mathrm{g})$ and THF $(10 \mathrm{x} \mathrm{X}$ $\mathrm{mL}$ ) were added to remove an excess of $\mathbf{2 a}$. The mixture was agitated at room temperature for $2 \mathrm{~h}$. The mixture was filtered and thoroughly washed with $\mathrm{CHCl}_{3}$. The 
filtrate was concentrated in vacuo and the residue was purified by preparative TLC to yield 2,3-disubstituted- $1 H$-inden-1-ones 7.

Table 2S

\begin{tabular}{|c|c|c|c|c|}
\hline entry & \multicolumn{1}{|c|}{$\begin{array}{c}\mathbf{3} \\
(\mathrm{mg}, \mathrm{mmol})\end{array}$} & $\begin{array}{c}\mathbf{2 a} \\
(\mathrm{mg}, \mathrm{mmol})\end{array}$ & $\begin{array}{c}\mathbf{1 0 c} \\
(\mathrm{mg}, \mu \mathrm{mol})\end{array}$ & $\begin{array}{c}\mathbf{7} \\
(\mathrm{mg}, \mathrm{mmol}, \%)\end{array}$ \\
\hline 1 & $\mathbf{3 b} ; 23.5,0.0986$ & $22.0,0.122$ & $3.7,5.1$ & $\mathbf{7 b} ; 33.1,0.0967,98$ \\
\hline 2 & $\mathbf{3 c} ; 25.9,0.0987$ & $22.2,0.123$ & $4.0,5.5$ & $\mathbf{7 c} ; 30.4,0.0830,84$ \\
\hline 3 & $\mathbf{3 d} ; 10.9,0.0989$ & $22.2,0.123$ & $3.9,5.3$ & $\mathbf{7 d} ; 17.5,0.0817,83$ \\
\hline 4 & $\mathbf{3 e} ; 16.7,0.102$ & $23.0,0.128$ & $3.8,5.2$ & $\mathbf{7 e} ; 15.9,0.0592,58$ \\
& & & & $\mathbf{6 e} ; 4.3,0.0151,15$ \\
\hline 5 & $\mathbf{3 f} ; 59.2,0.510$ & $110.3,0.612$ & $18.3,25.0$ & $\mathbf{7 f} ; 77.2,0.350,69$ \\
& & & $\mathbf{7 f} ; 15.4,0.0699,14$ \\
\hline 6 & $\mathbf{3 g} ; 16.0,0.0986$ & $22.6,0.126$ & $3.6,4.9$ & $\mathbf{7 g} ; 8.1,0.0304,31$ \\
\hline
\end{tabular}

2,3-Di(4-methoxyphenyl)-1H-inden-1-one ${ }^{17}$ (7b) was isolated by preparative TLC eluting with $20 \% \mathrm{EtOAc/hexane} \mathrm{repeated} \mathrm{two} \mathrm{times.}$

Red solid. $\mathrm{R}_{f}=0.38$ (30\% EtOAc/hexane). Mp: $115-118^{\circ} \mathrm{C}$. IR (neat): $2931,1700,1603$, 1501, 1245, 1173, 1028, 820, $735 \mathrm{~cm}^{-1} .{ }^{1} \mathrm{H}$ NMR (400 M Hz, $\left.\mathrm{CDCl}_{3}\right): \delta 7.55$ (d, J=6.8 $\mathrm{Hz}, 1 \mathrm{H}), 7.37-7.33$ (m, 3H), 7.27-7.22 (m, 3H), 7.16 (d, J=7.3 Hz, 1H), 6.93 (d, J=8.5 $\mathrm{Hz}, 2 \mathrm{H}), 6.82(\mathrm{~d}, J=9.0 \mathrm{~Hz}, 2 \mathrm{H}), 3.85$ (s, 3H), 3.79 (s, 3H). ${ }^{13} \mathrm{C}$ NMR (100 M Hz, $\left.\mathrm{CDCl}_{3}\right): \delta 196.6,160.0,158.8,153.5,145.3,133.1,131.10,131.06,130.8,130.0,128.4$, 125.0, 123.3, 122.6, 120.8, 114.1, 113.5, 55.3, 55.2. LRMS (EI) $\mathrm{m} / \mathrm{z}$ (relative intensity) $342[\mathrm{M}]^{+}$(100), 327 (23), 171 (9.9). HRMS (EI, [M] $]^{+}$): calcd for $\mathrm{C}_{23} \mathrm{H}_{18} \mathrm{O}_{3}, 342.1256$; found, 342.1238 .

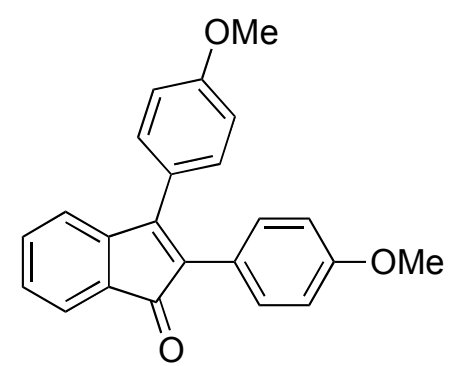

2,3-Di(4-acetylphenyl)-1H-inden-1-one (7c) was isolated by preparative TLC eluting with $10 \% \mathrm{EtOAc/toluene} \mathrm{repeated} \mathrm{four} \mathrm{times.}$

Orange solid. $\mathrm{R}_{f}=0.15$ (30\% EtOAc/hexane). Mp: 186-188 ${ }^{\circ} \mathrm{C}$. IR (neat): 3346, 3064, 
1715, 1684, 1600, 1356, 1262, 955, $716 \mathrm{~cm}^{-1} .{ }^{1} \mathrm{H}$ NMR (400 M Hz, $\left.\mathrm{CDCl}_{3}\right): \delta 8.02(\mathrm{~d}$, $J=8.5 \mathrm{~Hz}, 2 \mathrm{H}), 7.85$ (d, J=8.3 Hz, 2H), 7.63 (d, $J=7.1 \mathrm{~Hz}, 1 \mathrm{H}), 7.48$ (d, $J=8.5 \mathrm{~Hz}, 2 \mathrm{H})$, 7.42 (dd, J=7.6, 7.2 Hz, 1H), 7.37-7.33 (m, 3H), 7.13 (d, J=7.1 Hz, 1H), 2.64 (s, 3H), $2.58(\mathrm{~s}, 3 \mathrm{H}) .{ }^{13} \mathrm{C} \mathrm{NMR}\left(100 \mathrm{M} \mathrm{Hz}, \mathrm{CDCl}_{3}\right): \delta 197.4,197.0,195.2,155.3,144.3,137.6$, 137.0, 136.1, 135.1, 133.7, 132.0, 130.3, 130.0, 129.6, 128.8, 128.6, 128.0, 123.4, 121.4, 26.7, 26.6. LRMS (EI) $\mathrm{m} / \mathrm{z}$ (relative intensity) $366[\mathrm{M}]^{+}$(85), 351 (100), 281 (24), 252 (20), 168 (30). HRMS (EI, [M] $]^{+}$): calcd for $\mathrm{C}_{25} \mathrm{H}_{18} \mathrm{O}_{3}, 366.1256$; found, 366.1271.

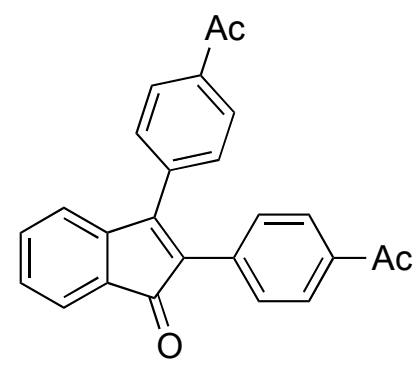

2,3-Dipropyl- $1 H$-inden-1-one ${ }^{18}$ (7d) was isolated by preparative TLC eluting with $1 \%$ EtOAc/toluene.

Yellow oil. $\mathrm{R}_{f}=0.61$ (30\% EtOAc/hexane). IR (neat): 2960, 2931, 2871, 1702, 1455, 762, $720 \mathrm{~cm}^{-1} .{ }^{1} \mathrm{H}$ NMR $\left(400 \mathrm{M} \mathrm{Hz}, \mathrm{CDCl}_{3}\right.$ ): $\delta 7.37$ (dd, $\left.J=7.1,1.1 \mathrm{~Hz}, 1 \mathrm{H}\right), 7.31$ (ddd, $J=7.7$, 7.2, $1.1 \mathrm{~Hz}, 1 \mathrm{H}), 7.15(\mathrm{dd}, J=7.7,7.1 \mathrm{~Hz}, 1 \mathrm{H}), 7.02$ (d, $J=7.2 \mathrm{~Hz}, 1 \mathrm{H}), 2.52$ (t, $J=7.7 \mathrm{~Hz}$, 2H), 2.24 (t, $J=7.7 \mathrm{~Hz}, 2 \mathrm{H}), 1.65$ (tq, $J=7.7,7.3 \mathrm{~Hz}, 2 \mathrm{H}), 1.50$ (tq, $J=7.7,7.3 \mathrm{~Hz}, 2 \mathrm{H})$, $1.03(\mathrm{t}, J=7.3 \mathrm{~Hz}, 3 \mathrm{H}), 0.94$ (t, $J=7.3 \mathrm{~Hz}, 3 \mathrm{H}) .{ }^{13} \mathrm{C} \mathrm{NMR}\left(100 \mathrm{M} \mathrm{Hz}, \mathrm{CDCl}_{3}\right): \delta 198.2$, 157.4, 145.5, 134.7, 133.0, 131.0, 127.7, 121.5, 118.9, 28.4, 25.0, 22.7, 21.4, 14.6, 14.3. LRMS (EI) $m / z$ (relative intensity) $214[\mathrm{M}]^{+}$(67), 185 (100), 171 (36), 157 (26), 143 (56), 128 (19), 115 (21). HRMS (EI, [M] $]^{+}$): calcd for $\mathrm{C}_{15} \mathrm{H}_{18} \mathrm{O}, 214.1358$; found, 214.1339.<smiles>CCCC1=C(CCC)c2ccccc2C1=O</smiles>

$5,6,7,8,9,10,11,12,13,14$-Decahydrocyclododeca $[a]-15 H$-inden-15-one ${ }^{19} \quad(\mathbf{7 e}) \quad$ and 7,8,9,10,11,12,13,14,15,16-Decahydro-6-oxa-cyclododeca[a]-naphthalen-5-one $\quad(\mathbf{6 e})$ were separated by preparative TLC eluting with $10 \%$ EtOAc/hexane repeated three times.

7e as the first effluent.

Yellow solid. $\mathrm{R}_{f}=0.61$ (30\% EtOAc/hexane). Mp: 77-80 ${ }^{\circ} \mathrm{C}$. IR (neat): 2929, 2852, 1698, 
1590, 1457, 940, 766, $726 \mathrm{~cm}^{-1} .{ }^{1} \mathrm{H}$ NMR (400 M Hz, $\mathrm{CDCl}_{3}$ ): $\delta 7.37$ (dd, J=7.0, $1.2 \mathrm{~Hz}$, 1H), 7.31 (ddd, $J=7.1,7.8,1.2 \mathrm{~Hz}, 1 \mathrm{H}), 7.15$ (ddd, J=7.0, 7.8, $1.0 \mathrm{~Hz}, 1 \mathrm{H}), 7.06$ (dd, $J=7.1,1.0 \mathrm{~Hz}, 1 \mathrm{H}), 2.58(\mathrm{t}, J=7.2 \mathrm{~Hz}, 2 \mathrm{H}), 2.30(\mathrm{t}, J=7.0 \mathrm{~Hz}, 2 \mathrm{H}), 1.83-1.76(\mathrm{~m}, 2 \mathrm{H})$, 1.69-1.63 (m, 2H), 1.50-1.25 (m, 12H). ${ }^{13} \mathrm{C}$ NMR (100 M Hz, $\left.\mathrm{CDCl}_{3}\right)$ : $\delta$ 198.5, 157.7, 145.5, 134.9, 133.0, 131.1, 127.7, 121.4, 119.4, 26.6, 25.8, 25.4, 25.1, 24.7, 24.5, 23.7, 22.4, 22.2, 20.7. LRMS (EI) $\mathrm{m} / z$ (relative intensity) $268[\mathrm{M}]^{+}$(100), 185 (31), 157 (65), 144 (58), 128 (29). HRMS (EI, [M] $]^{+}$): calcd for $\mathrm{C}_{19} \mathrm{H}_{24} \mathrm{O}, 268.1827$; found, 268.1807.

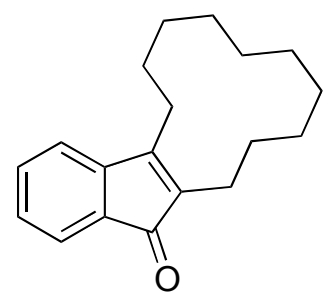

6e as the second effluent.

White solid. $\mathrm{R}_{f}=0.53$ (30\% EtOAc/hexane). Mp: 123-126 ${ }^{\circ} \mathrm{C}$. IR (neat): 2929, 2863, 1727, 1717, 1630, 1470, 1084, 776, $714 \mathrm{~cm}^{-1} .{ }^{1} \mathrm{H}$ NMR (400 M Hz, $\left.\mathrm{CDCl}_{3}\right): \delta 8.32(\mathrm{dd}$, $J=8.1,1.0 \mathrm{~Hz}, 1 \mathrm{H}), 7.72$ (ddd, $J=8.1,7.3,1.0 \mathrm{~Hz}, 1 \mathrm{H}), 7.57$ (d, $J=8.1 \mathrm{~Hz}, 1 \mathrm{H}), 7.46$ (dd, $J=8.1,7.3 \mathrm{~Hz}, 1 \mathrm{H}), 2.70(\mathrm{t}, J=7.1 \mathrm{~Hz}, 2 \mathrm{H}), 2.63(\mathrm{t}, J=7.6 \mathrm{~Hz}, 2 \mathrm{H}), 1.92-1.85(\mathrm{~m}, 2 \mathrm{H})$, 1.76-1.70 (m, 2H), 1.55-1.33 (m, 12H). ${ }^{13} \mathrm{C} \mathrm{NMR}\left(100 \mathrm{M} \mathrm{Hz}, \mathrm{CDCl}_{3}\right)$ : $\delta 162.4,154.4$, 137.7, 134.0, 129.4, 126.7, 122.9, 120.6, 112.1, 27.6, 27.1, 25.2, 25.1, 25.0, 25.0, 23.8, 23.6, 22.8, 21.9. LRMS (EI) $m / z$ (relative intensity) $284[\mathrm{M}]^{+}(100), 172$ (19). HRMS (EI, [M] $]^{+}$): calcd for $\mathrm{C}_{19} \mathrm{H}_{24} \mathrm{O}_{2}, 284.1776$; found, 284.1755 .

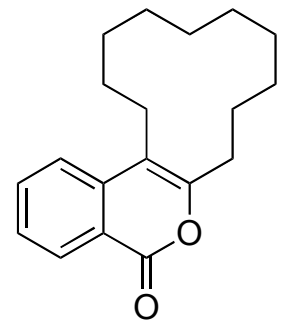

3-Methyl-2-phenyl-1H-inden-1-one ${ }^{20}$ (7f) and 2-methyl-3-phenylinden-1-one ${ }^{20} \quad$ (7f') were separated by preparative TLC eluting with $10 \%$ EtOAc/hexane repeated four times. The first effluent including 7f' was further purified by preparative TLC eluting with $1 \%$ EtOAc/toluene repeated three times.

7f as the second effluent.

Orange solid. $\mathrm{R}_{f}=0.53$ (30\% EtOAc/hexane). Mp: $61-65^{\circ} \mathrm{C}$. IR (neat): 3060, 1696, 1598, 1465, 1378, 853, 753, $699 \mathrm{~cm}^{-1} .{ }^{1} \mathrm{H}$ NMR (400 M Hz, $\left.\mathrm{CDCl}_{3}\right): \delta 7.49$ (d, J=7.1 Hz, 1H), 7.46-7.40 (m, 5H), 7.37-7.32 (m, 1H), 7.26 (dd, J=6.8, 7.1 Hz, 1H), 7.17 (d, J=7.3 Hz, 
1H), 2.33 (s, 3H). $\left.{ }^{13} \mathrm{C} \mathrm{NMR} \mathrm{(100} \mathrm{M} \mathrm{Hz,} \mathrm{CDCl}_{3}\right): \delta 196.1,154.4,145.7,133.4,133.3$, 131.0, 130.3, 129.4, 128.7, 128.1, 127.5, 122.0, 119.3, 12.7. LRMS (EI) $\mathrm{m} / \mathrm{z}$ (relative intensity) $220[\mathrm{M}]^{+}$(100), 219 (39), 191 (37), 189 (19), 165 (12). HRMS (EI, [M] $]^{+}$): calcd for $\mathrm{C}_{16} \mathrm{H}_{12} \mathrm{O}, 220.0888$; found, 220.0875 .

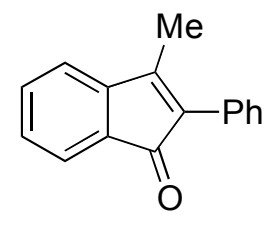

7f' as the first effluent.

Yellow solid. $\mathrm{R}_{f}=0.58$ (30\% EtOAc/hexane). Mp: 76-81 ${ }^{\circ} \mathrm{C}$. IR (neat): 2943, 1698, 1605, 1451, 940, 774, 712, $700 \mathrm{~cm}^{-1} .{ }^{1} \mathrm{H}$ NMR (400 M Hz, $\left.\mathrm{CDCl}_{3}\right): \delta$ 7.54-7.43 (m, 6H), 7.30 (ddd, $J=7.8,7.4,1.2 \mathrm{~Hz}, 1 \mathrm{H}), 7.19$ (dd, $J=7.8,6.8 \mathrm{~Hz}, 1 \mathrm{H}), 7.06$ (d, J=7.3 Hz, 1H), 1.93 (s, 3H). ${ }^{13} \mathrm{C}$ NMR (100 M Hz, $\left.\mathrm{CDCl}_{3}\right): \delta 197.8,154.3,145.3,132.8,132.4,130.8,130.7$, 128.8, 128.4, 127.74, 127.69, 122.2, 120.1, 8.6. LRMS (EI) $\mathrm{m} / z$ (relative intensity) 220 $[\mathrm{M}]^{+}$(100), 219 (78), 191 (41), 189 (25), 165 (16). HRMS (EI, [M] $]^{+}$: calcd for $\mathrm{C}_{16} \mathrm{H}_{12} \mathrm{O}$, 220.0888; found, 220.0875 .<smiles>CC1=C(c2ccccc2)c2ccccc2C1=O</smiles>

3-tert-Butyl-2-(tert-butylethynyl)inden-1-one ${ }^{21}$ (7g) was isolated by preparative TLC eluting with $1 \%$ EtOAc/toluene repeated three times.

Orange oil. $\mathrm{R}_{f}=0.63$ (30\% EtOAc/hexane). IR (neat): 2968, 1717, 1459, 1256, 1202, 768, $726 \mathrm{~cm}^{-1} .{ }^{1} \mathrm{H}$ NMR (400 M Hz, $\mathrm{CDCl}_{3}$ ): $\delta$ 7.45-7.42 (m, 2H), 7.34 (ddd, J=7.8, 7.6, 1.4 $\mathrm{Hz}, 1 \mathrm{H}), 7.19$ (ddd, $J=7.8,7.6,0.7 \mathrm{~Hz}, 1 \mathrm{H}), 1.53$ (s, 9H), 1.31 (s, 9H). ${ }^{13} \mathrm{C}$ NMR (100 M $\left.\mathrm{Hz}, \mathrm{CDCl}_{3}\right)$ : $\delta 194.4,168.4,144.6,133.2,130.9,128.1,123.5,122.4,117.3,110.4,72.4$, 36.7, 30.7, 29.7, 28.7. LRMS (EI) $\mathrm{m} / z$ (relative intensity) $266[\mathrm{M}]^{+}$(100), 251 (87), 236 (78), 223 (63), 209 (51), 195 (40), 165 (24). HRMS (EI, [M] $]^{+}$): calcd for $\mathrm{C}_{19} \mathrm{H}_{22} \mathrm{O}$, 266.1671; found, 266.1667.<smiles>CC(C)(C)C#CC1=C(C(C)(C)C)c2ccccc2C1=O</smiles> 


\section{Pd(II)-Catalyzed Synthesis of 2-Naphthols 15a (Scheme 4):}

To a test tube containing $\mathbf{3 b}(23.1 \mathrm{mg}, 0.0969 \mathrm{mmol}), \mathbf{1 4 a}(33.2 \mathrm{mg}, 0.120 \mathrm{mmol})$, and 10c $(3.7 \mathrm{mg}, 5.1 \mu \mathrm{mol})$ was added anhydrous $\mathrm{MeOH}(0.5 \mathrm{~mL})$ under argon. The resulting mixture was sealed with a screw cap and agitated at $80^{\circ} \mathrm{C}$ for $2 \mathrm{~h}$. The mixture was cooled down to room temperature, and then PS-DEAM ${ }^{\mathrm{TM}}(1.63 \mathrm{mmol} / \mathrm{g}, 0.15 \mathrm{~g}$, $0.24 \mathrm{mmol})$ and THF (2 mL) were added to remove an excess of 14a. The mixture was agitated at room temperature for $2 \mathrm{~h}$. The mixture was filtered and thoroughly washed with $\mathrm{CHCl}_{3}$. The filtrate was concentrated in vacuo and the residue was purified by preparative TLC eluting with $1 \%$ EtOAc/toluene repeated four times to yield 3,4-di(4-methoxyphenyl)-2-naphthol (15a) (28.6 mg, $0.0802 \mathrm{mmol}, 83 \%$ ).

White solid. $\mathrm{R}_{f}=0.32$ (30\% EtOAc/hexane). Mp: 199-204 ${ }^{\circ} \mathrm{C}$. IR (neat): 3458, 2833, $1609,1513,1245,1229,1175,1027,816,754 \mathrm{~cm}^{-1} .{ }^{1} \mathrm{H}$ NMR $\left(400 \mathrm{M} \mathrm{Hz}, \mathrm{CDCl}_{3}\right): \delta$ $7.76(\mathrm{~d}, J=8.3 \mathrm{~Hz}, 1 \mathrm{H}), 7.49$ (d, $J=8.5 \mathrm{~Hz}, 1 \mathrm{H}), 7.42$ (dd, $J=8.3,7.6 \mathrm{~Hz}, 1 \mathrm{H}), 7.38$ (s, 1H), 7.21 (dd, $J=8.5,7.6 \mathrm{~Hz}, 1 \mathrm{H}), 7.07$ (d, J=8.5 Hz, 2H), 7.03 (d, $J=8.5 \mathrm{~Hz}, 2 \mathrm{H}), 6.82$ $(\mathrm{d}, J=8.5 \mathrm{~Hz}, 2 \mathrm{H}), 6.78(\mathrm{~d}, J=8.5 \mathrm{~Hz}, 2 \mathrm{H}), 5.06(\mathrm{~s}, 1 \mathrm{H}), 3.79(\mathrm{~s}, 3 \mathrm{H}), 3.78(\mathrm{~s}, 3 \mathrm{H}) .{ }^{13} \mathrm{C}$ NMR (100 M Hz, $\left.\mathrm{CDCl}_{3}\right)$ : $\delta 159.0,158.2,151.0,140.0,134.4,132.1,131.9,131.0$, $129.5,128.5,126.97,126.95,126.5,126.2,123.5,114.3,113.1,109.0,55.15,55.12$. LRMS (EI) $\mathrm{m} / z$ (relative intensity) $356[\mathrm{M}]^{+}(100)$. HRMS (EI, [M] $]^{+}$): calcd for $\mathrm{C}_{24} \mathrm{H}_{20} \mathrm{O}_{3}, 356.1412$; found, 356.1400 .<smiles>COc1ccc(-c2c(O)cc3ccccc3c2-c2ccc(OC)cc2)cc1</smiles>

\section{Pd(II)-Catalyzed Synthesis of 2-Naphthols 15b (Scheme 4):}

To a test tube containing $3 \mathbf{b}(23.4 \mathrm{mg}, 0.0982 \mathrm{mmol}), \mathbf{1 4 b}(40.0 \mathrm{mg}, 0.120 \mathrm{mmol})$, and 10c $(3.7 \mathrm{mg}, 5.1 \mu \mathrm{mol})$ was added anhydrous $\mathrm{MeOH}(0.5 \mathrm{~mL})$ under argon. The resulting mixture was sealed with a screw cap and agitated at $80{ }^{\circ} \mathrm{C}$ for $2 \mathrm{~h}$. The mixture was cooled down to room temperature, and then PS-DEAM ${ }^{\mathrm{TM}}(1.63 \mathrm{mmol} / \mathrm{g}, 0.15 \mathrm{~g}$, $0.24 \mathrm{mmol})$ and THF ( $2 \mathrm{~mL}$ ) were added to remove an excess of $\mathbf{1 4 \mathbf { b }}$. The mixture was agitated at room temperature for $2 \mathrm{~h}$. The mixture was filtered and thoroughly washed with $\mathrm{CHCl}_{3}$. The filtrate was concentrated in vacuo and the residue was purified by 
preparative TLC eluting with $1 \%$ EtOAc/toluene repeated four times to yield methyl 2-hydroxy-3,4-di(4-methoxyphenyl)-1-naphthalenecarboxylate (15b) (38.1 mg, 0.0919 mmol, 94\%).

White solid. $\mathrm{R}_{f}=0.39$ (30\% EtOAc/hexane). Mp: 227-234 ${ }^{\circ} \mathrm{C}$. IR (neat): 2956, 1505 , $1345,1246,1229,1177,1152,1028,824,754 \mathrm{~cm}^{-1} .{ }^{1} \mathrm{H}$ NMR $\left(400 \mathrm{M} \mathrm{Hz}, \mathrm{CDCl}_{3}\right): \delta$ 12.55 (s, 1H), 8.79 (d, J=9.0 Hz, 1H), 7.55-7.52 (m, 2H), 7.25 (dd, J=8.1, 7.3 Hz, 1H), 7.02 (d, J=8.0 Hz, 2H), 7.00 (d, J=8.3 Hz, 2H), 6.79 (d, J=8.3 Hz, 2H), 6.76 (d, J=8.0 $\mathrm{Hz}, 2 \mathrm{H}), 4.13$ (s, 3H), 3.79 (s, 3H), 3.76 (s, 3H). ${ }^{13} \mathrm{C} \mathrm{NMR}\left(100 \mathrm{M} \mathrm{Hz}, \mathrm{CDCl}_{3}\right.$ ): $\delta 172.8$, 162.0, 158.2, 157.9, 146.9, 131.6, 131.4, 131.22, 131.18, 130.4, 128.5, 128.4, 128.0, 127.8, 124.9, 123.4, 113.1, 113.0, 104.2, 55.2, 55.1, 52.6. LRMS (EI) $\mathrm{m} / z$ (relative intensity) $414[\mathrm{M}]^{+}$(100), 382 (100), 354 (23), 311 (15), 239 (13), 191 (19). HRMS (EI, $\left.[\mathrm{M}]^{+}\right)$: calcd for $\mathrm{C}_{26} \mathrm{H}_{22} \mathrm{O}_{5}, 414.1467$; found, 414.1464.<smiles>COc1ccc(-c2c(O)c(C(C)=O)c3ccccc3c2-c2ccc(OC)cc2)cc1</smiles>

\section{Pd(II)-Catalyzed Synthesis of 2-Naphthols 15c (Scheme 4):}

To a test tube containing $\mathbf{3 b}(23.7 \mathrm{mg}, 0.0995 \mathrm{mmol}), \mathbf{1 4 c}(55.6 \mathrm{mg}, 0.129 \mathrm{mmol})$, and 10c $(3.8 \mathrm{mg}, 5.2 \mu \mathrm{mol})$ was added anhydrous $\mathrm{MeOH}(0.5 \mathrm{~mL})$ under argon. The resulting mixture was sealed with a screw cap and agitated at $80{ }^{\circ} \mathrm{C}$ for $2 \mathrm{~h}$. The mixture was cooled down to room temperature, and then PS-DEAM ${ }^{\mathrm{TM}}(1.63 \mathrm{mmol} / \mathrm{g}, 0.15 \mathrm{~g}$, $0.24 \mathrm{mmol})$ and THF ( $2 \mathrm{~mL})$ were added to remove an excess of 14c. The mixture was agitated at room temperature for $2 \mathrm{~h}$. The mixture was filtered and thoroughly washed with $\mathrm{CHCl}_{3}$. The filtrate was concentrated in vacuo and the residue was purified by recrystallization from to EtOAc yield 3,4-di(4-methoxyphenyl)-1-[(4-methylphenyl)sulfonyl]-2-naphthol (15c) (39.5 mg, $0.0774 \mathrm{mmol}, 78 \%$ ).

White solid. $\mathrm{R}_{f}=0.30$ (30\% EtOAc/hexane). Mp: 265-268 ${ }^{\circ} \mathrm{C}$. IR (neat): 3162, 2956, 2836, 1509, 1405, 1287, 1245, 1160, 1117, 1027, 774, $722 \mathrm{~cm}^{-1} .{ }^{1} \mathrm{H}$ NMR (400 M Hz, $\left.\mathrm{CDCl}_{3}\right): \delta 11.55(\mathrm{~s}, 1 \mathrm{H}), 8.44(\mathrm{~d}, J=8.5 \mathrm{~Hz}, 1 \mathrm{H}), 7.93(\mathrm{~d}, J=8.3 \mathrm{~Hz}, 2 \mathrm{H}), 7.49$ (d, J=8.2 $\mathrm{Hz}, 1 \mathrm{H}), 7.44$ (dd, J=8.5, 7.4 Hz, 1H), 7.32 (d, J=8.3 Hz, 2H), 7.21 (dd, J=8.2, 7.4 Hz, 
1H), 7.04 (d, J=8.2 Hz, 2H), 6.97 (d, J=8.2 Hz, 2H), 6.78 (d, J=8.2 Hz, 2H), 6.78 (d, $J=8.2 \mathrm{~Hz}, 2 \mathrm{H}), 3.78$ (s, 3H), 3.77 (s, 3H), 2.40 (s, 3H). ${ }^{13} \mathrm{C} \mathrm{NMR}\left(100 \mathrm{M} \mathrm{Hz}, \mathrm{CDCl}_{3}\right): \delta$ 158.3, 158.0, 156.7, 147.8, 144.4, 139.2, 132.0, 131.7, 131.3, 129.8, 129.7, 128.9, 128.6, 128.2, 128.0, 127.9, 126.6, 124.0, 122.6, 113.1, 113.0, 111.4, 55.2, 55.1, 21.8. LRMS (EI) $\mathrm{m} / z$ (relative intensity) $510[\mathrm{M}]^{+}(100)$. HRMS (EI, [M] $]^{+}$): calcd for $\mathrm{C}_{31} \mathrm{H}_{26} \mathrm{O}_{5} \mathrm{~S}$, 510.1501; found, 510.1489.<smiles>COc1ccc(-c2c(O)c(F)c3ccccc3c2-c2ccc(OC)cc2)cc1</smiles>

\section{Pd(0)-Catalyzed Annulation of 3a with 1a (Scheme 5):}

To a test tube containing 1a $(33.3 \mathrm{mg}, 0.127 \mathrm{mmol}), 3 \mathbf{3 a}(17.9 \mathrm{mg}, 0.100 \mathrm{mmol})$, $\operatorname{PdCp}\left(\eta^{3}-\mathrm{C}_{3} \mathrm{H}_{5}\right)(11.0 \mathrm{mg}, 0.0517 \mathrm{mmol})$, and dppe $(20.0 \mathrm{mg}, 0.0502 \mathrm{mmol})$ were added anhydrous $\mathrm{MeOH}(0.5 \mathrm{~mL})$ and $\mathrm{Et}_{3} \mathrm{~N}(33 \mu \mathrm{L}, 0.24 \mathrm{mmol})$ under argon. The resulting mixture was sealed with a screw cap and agitated at $80{ }^{\circ} \mathrm{C}$ for $24 \mathrm{~h}$. The mixture was cooled down to room temperature, and then partitioned between $\mathrm{Et}_{2} \mathrm{O}$ and saturated aqueous $\mathrm{NH}_{4} \mathrm{Cl}$. The aqueous layer was extracted with $\mathrm{Et}_{2} \mathrm{O}$ twice. The combined organic layers were washed with water and brine, dried over $\mathrm{MgSO}_{4}$, and concentrated in vacuo. The residue was purified by preparative TLC eluting with $1 \%$ EtOAc/toluene repeated two times followed by GPC to yield $7 \mathbf{a}(5.9 \mathrm{mg}, 0.021 \mathrm{mmol}, 21 \%)$. 
References

1) (a) Baudoin, O.; Guénard, D.; Guéritte, F. J. Org. Chem. 2000, 65, 9268-9271. (b) Joncour, A.; Décor, A.; Thoret, S.; Chiaroni, A.; Baudoin, O. Angew. Chem. Int. Ed. 2006, $45,4149-4152$.

2) Zhdankin, V. V.; Koposov, A. Y.; Litvinov, D. N.; Ferguson, M. J.; McDonald, R.; Luu, T.; Tykwinski, R. R. J. Org. Chem. 2005, 70, 6484-6491.

3) Wolan, A.; Zaidlewicz, M. Org. Biomol. Chem. 2003, 1, 3274-3276.

4) (a) Zhu, L.; Duquette, J.; Zhang, M. J. Org. Chem. 2005, 70, 6484-6491. (b) Jones, S. B.; He, L.; Castle, S. L. Org. Lett. 2006, 8, 3757-3760.

5) Fong, C. W.; Lincoln, S. F.; Williams, E. H. Aust. J. Chem. 1978, 31, 2623-2628.

6) Reboredo, F. J.; Treus, M.; Estevez, J. C.; Castedo, L.; Estevez, R. J. Synlett 2003, 1603-1606.

7) Larock, R. C.; Fried, C. A. J. Am. Chem. Soc. 1990, 112, 5882-5884.

8) Costa, A.; Najera, C.; Sansano, J. M. J. Org. Chem. 2002, 67, 5216-5225.

9) (a) Zhang, Daohua; Yum, Eul Kgun; Liu, Zhijian; Larock, Richard C. Org. Lett. 2005, 7, 4963-4966. (b) Zhang, D.; Liu, Z.; Yum, E. K.; Larock, R. C. J. Org. Chem. 2007, $72,251-262$.

10) Sonogashira, K.; Tohda, Y.; Hagihara, N. Tetrahedron Lett. 1975, 4467-4470.

11) Mio, M. J.; Kopel, L. C.; Braun, J. B.; Gadzikwa, T. L.; Hull, K. L.; Brisbois, R. G.; Markworth C. J.; Grieco, P. A. Org. Lett. 2002, 4, 3199-3202.

12) Brummond, K. M.; Gesenberg, K. D.; Kent, J. L.; Kerekes, A. D. Tetrahedron Lett. 1998, 39, 8613-8616.

13) (a) Marson, A.; van Oort, A. B.; Mul W. P. Eur. J. Inorg. Chem. 2002, 3028-3031.

(b) Bianchini, C.; Meli, A.; Oberhauser, W. Organometallics 2003, 22, 4281-4285.

(c) Luo, H.-K.; Kou, Y.; Wang, X.-W.; Li, D.-G. J. Mol. Catal. A 2000, 151, 91-113.

(d) Davis, J. A.; Hartley, E. R.; Muray, S. G. J. Chem. Soc., Dalton Trans. 1979, 1705-1708. (e) Davis, J. A.; Hartley, E. R.; Muray, S. G. J. Chem. Soc., Dalton Trans. 1980, 2246-2249.

14) PS-DEAM ${ }^{\mathrm{TM}}$ was purchased from Biotage.

15) Chang, K.-J.; Rayabarapu, D. K.; Cheng, C.-H. J. Org. Chem. 2004, 69, 4781-4787.

16) Pouchhert, C.; Behnke, J. Aldrich Library of ${ }^{13} \mathrm{C}$ and ${ }^{1} H$ FT-NMR Spectra, Aldrich Chemical Co., 1992.

17) Pletnev, A. A.; Tian, Q.; Larock, R. C. J. Org. Chem. 2002, 67, 9276-9287. 
18) Larock, R. C.; Doty, M. J.; Cacchi, S. J. Org. Chem. 1993, 58, 4579-4583.

19) Harada, Y.; Nakanishi, J.; Fujihara, H.; Tobisu, M.; Fukumoto, Y.; Chatani, N. J. Am. Chem. Soc. 2007, 129, 5766-571.

20) Liebeskind, L. S.; South, M. S. J. Org. Chem. 1980, 45, 5426-5430.

21) Miura, T.; Murakami, M. Org. Lett. 2005, 7, 3339-3341. 


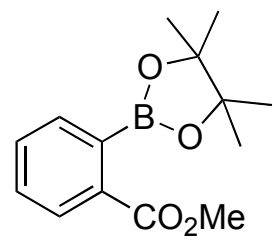

$2 b$
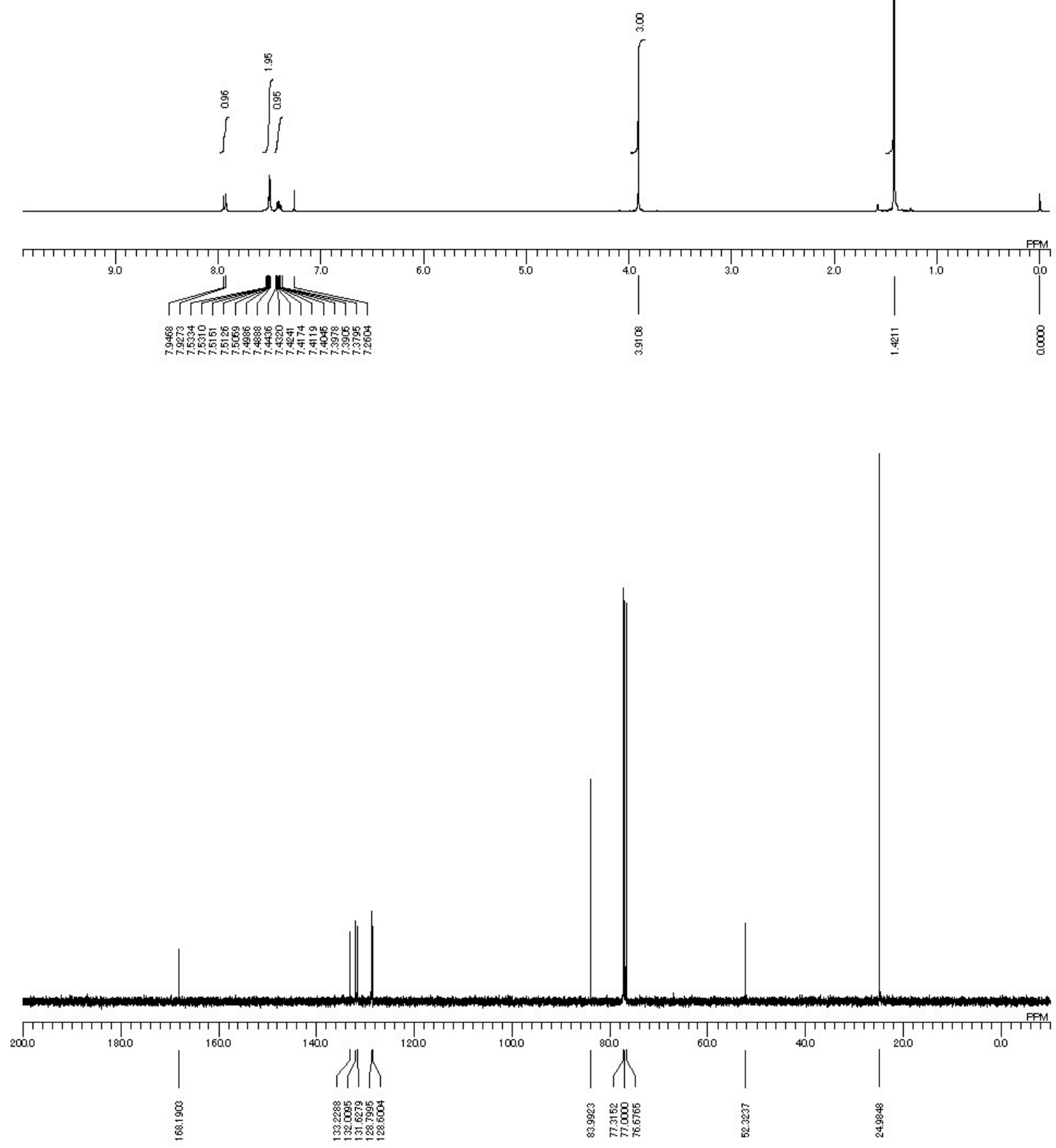


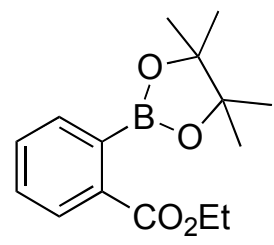

2c
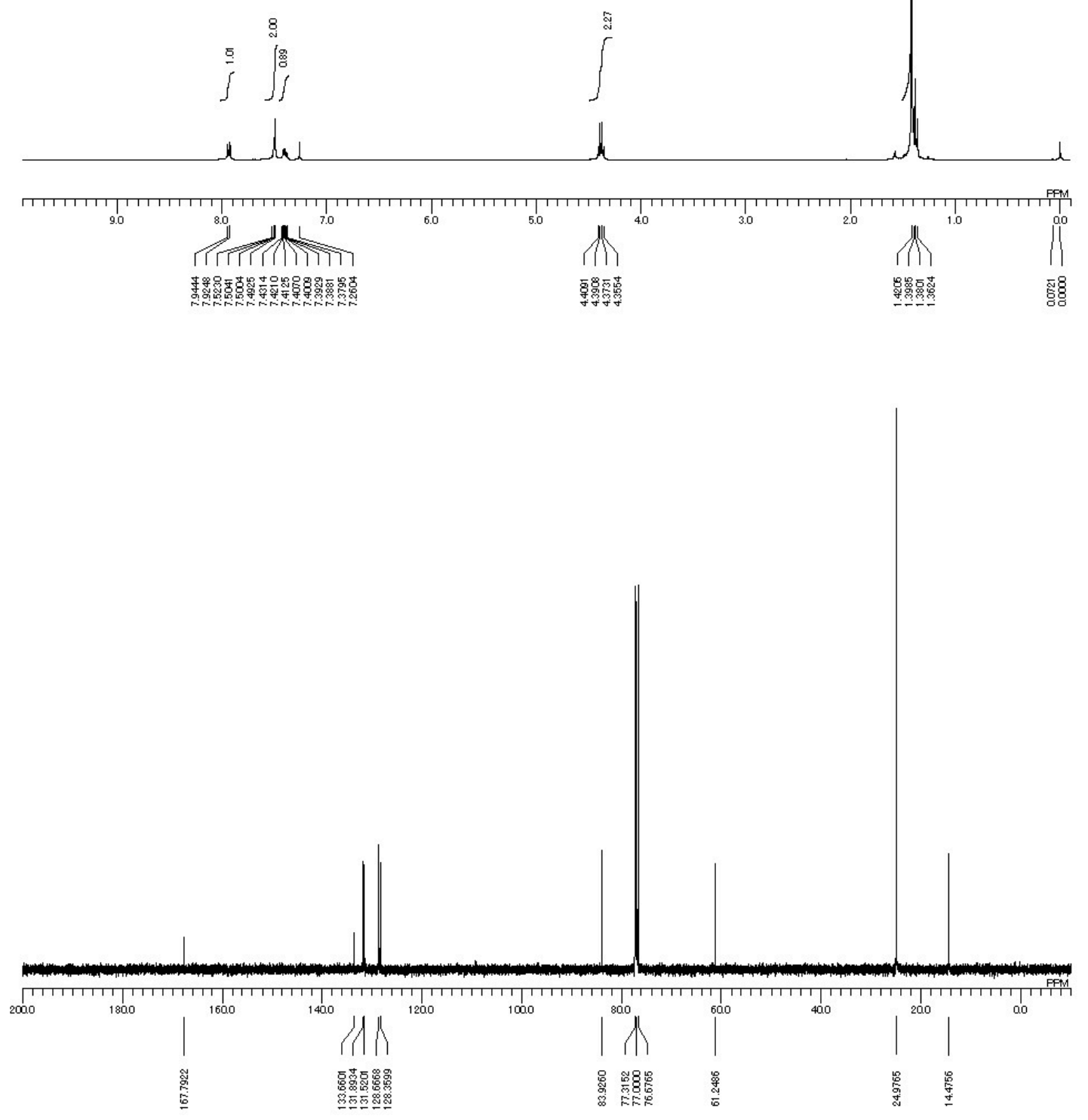


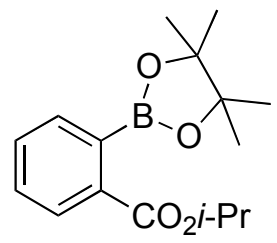

2d
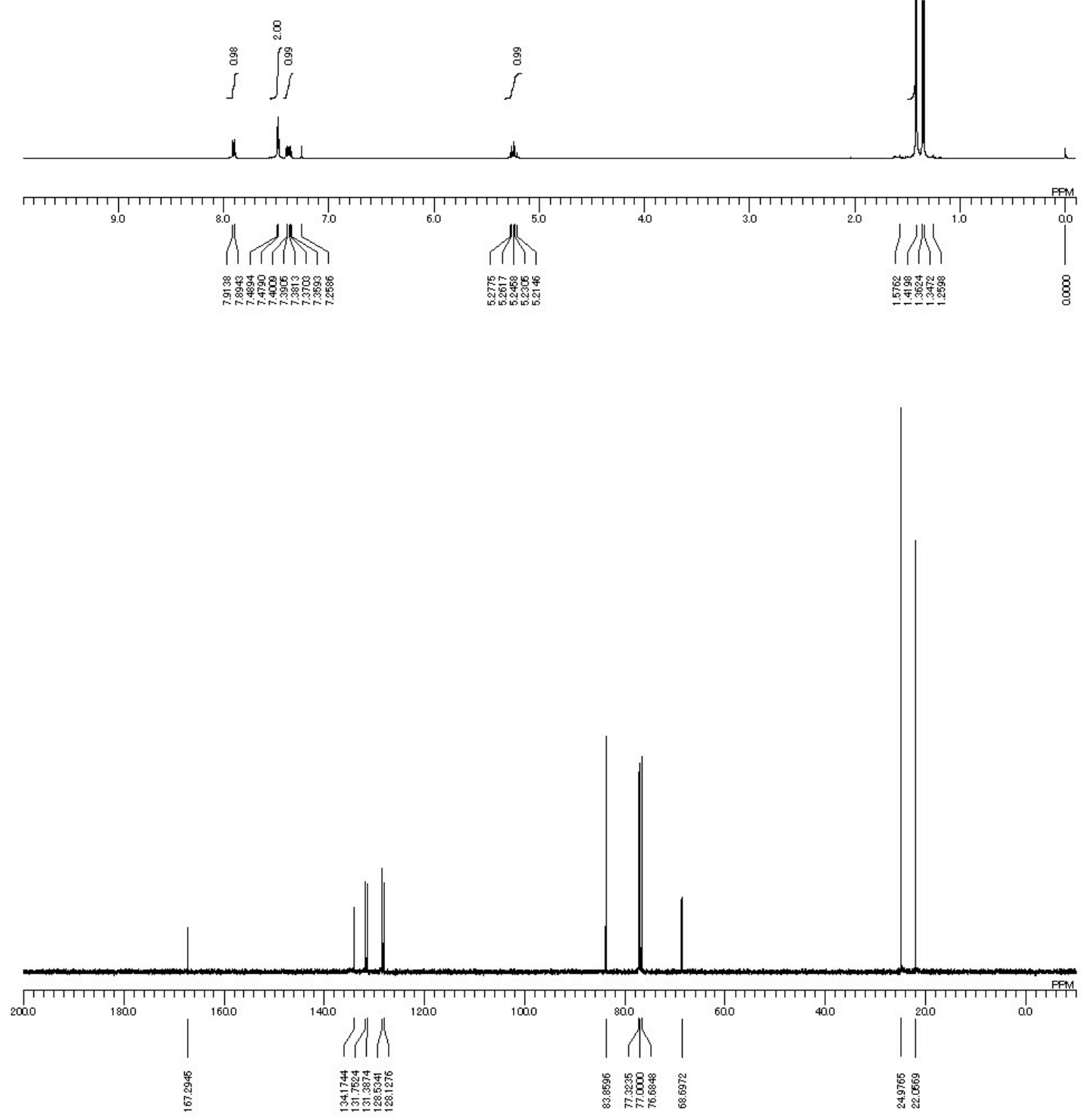

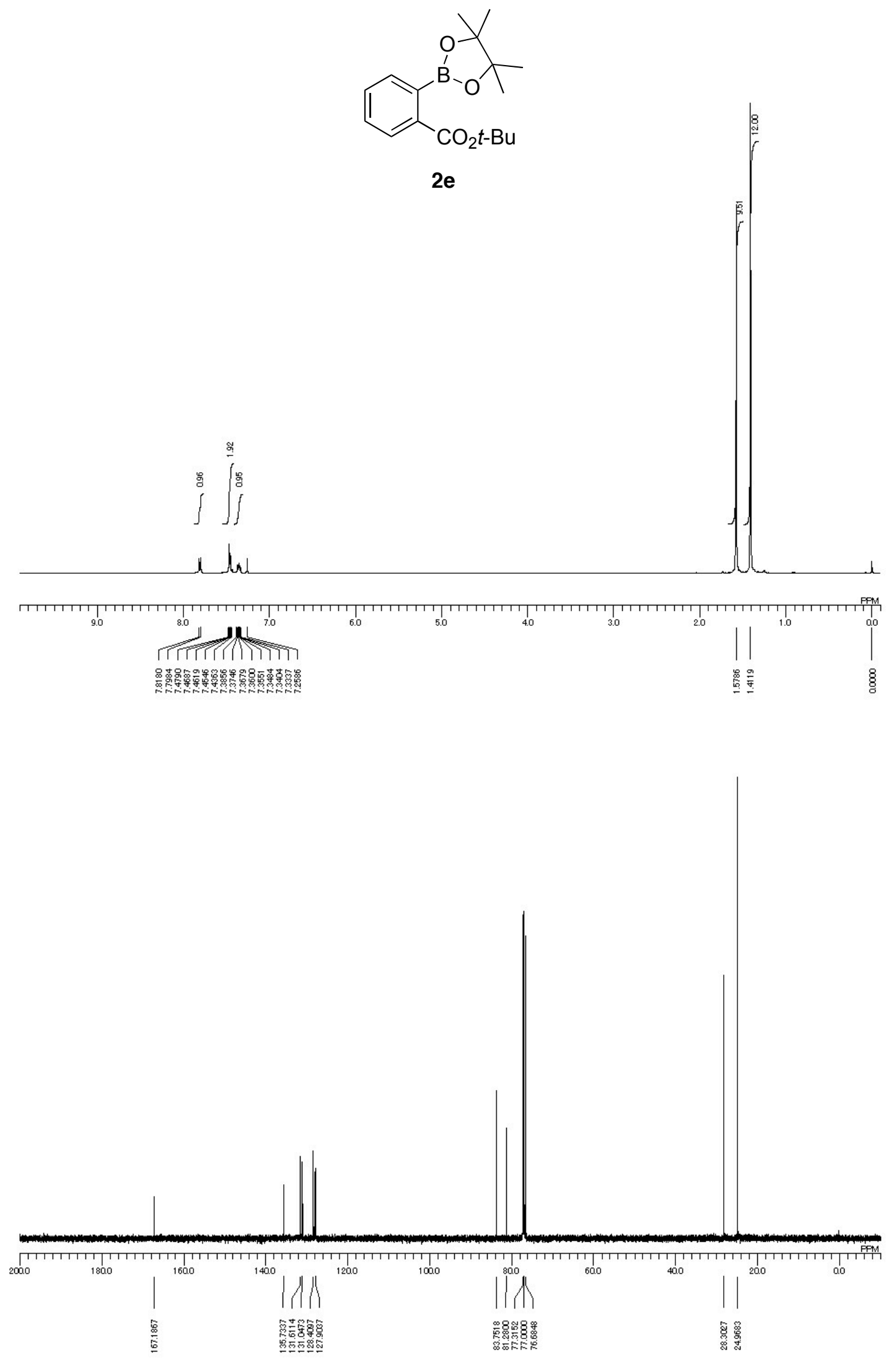


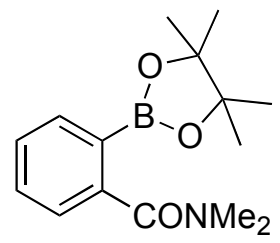

$2 f$
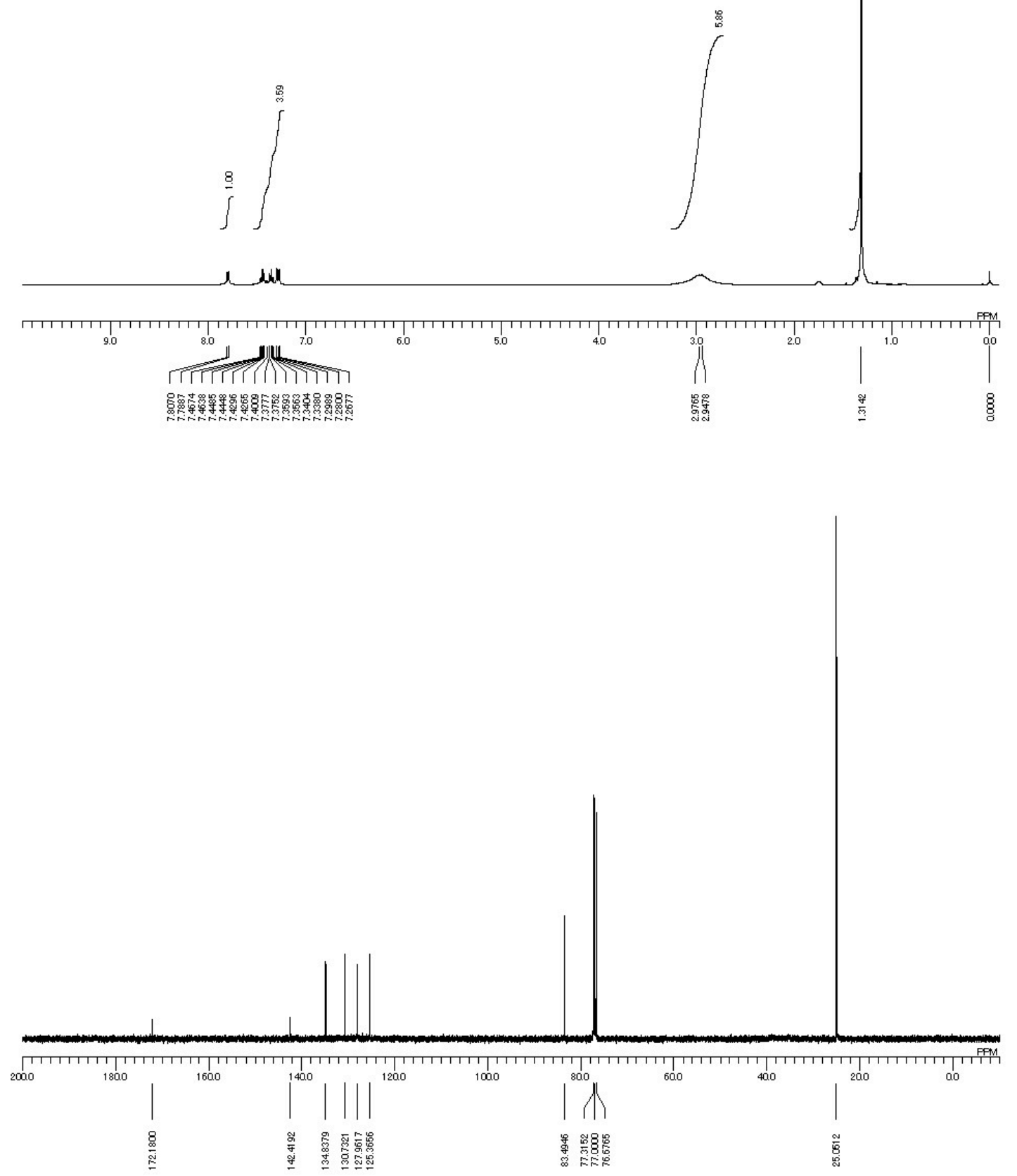


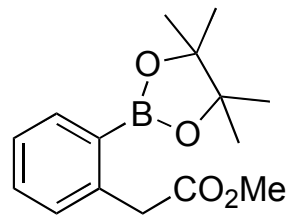

$14 a$
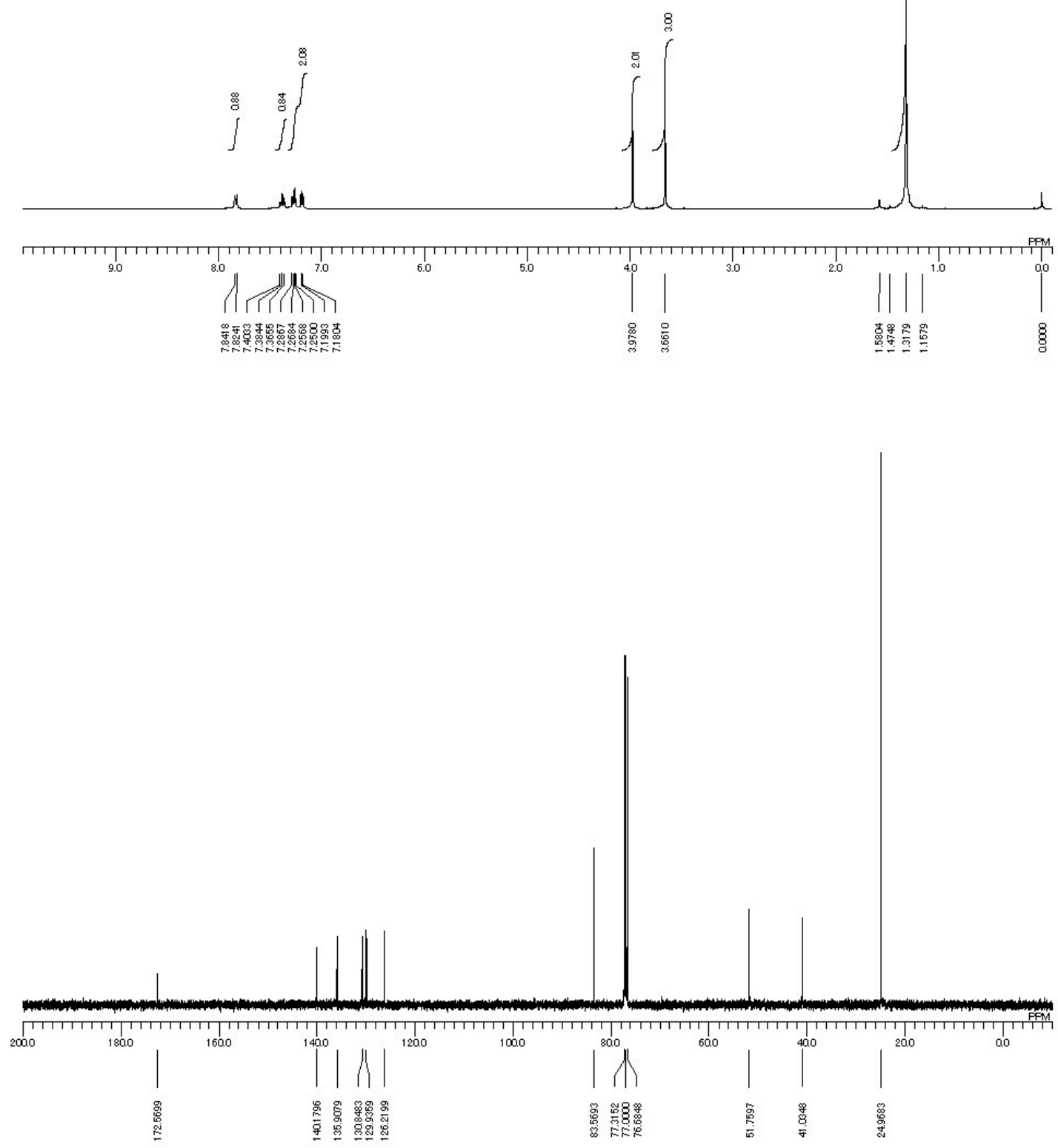

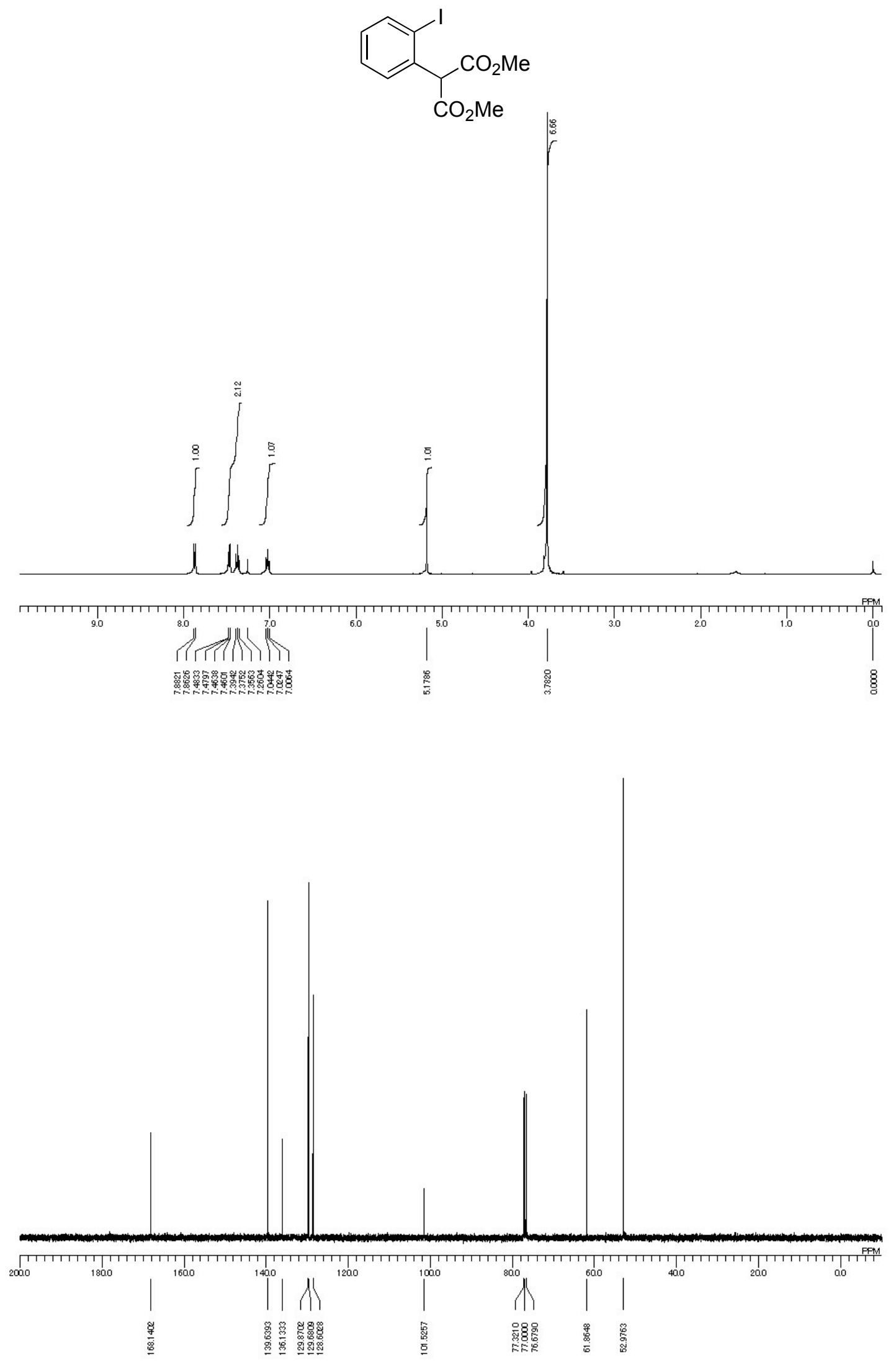


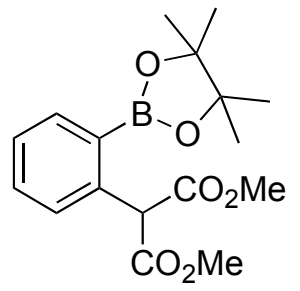

14b
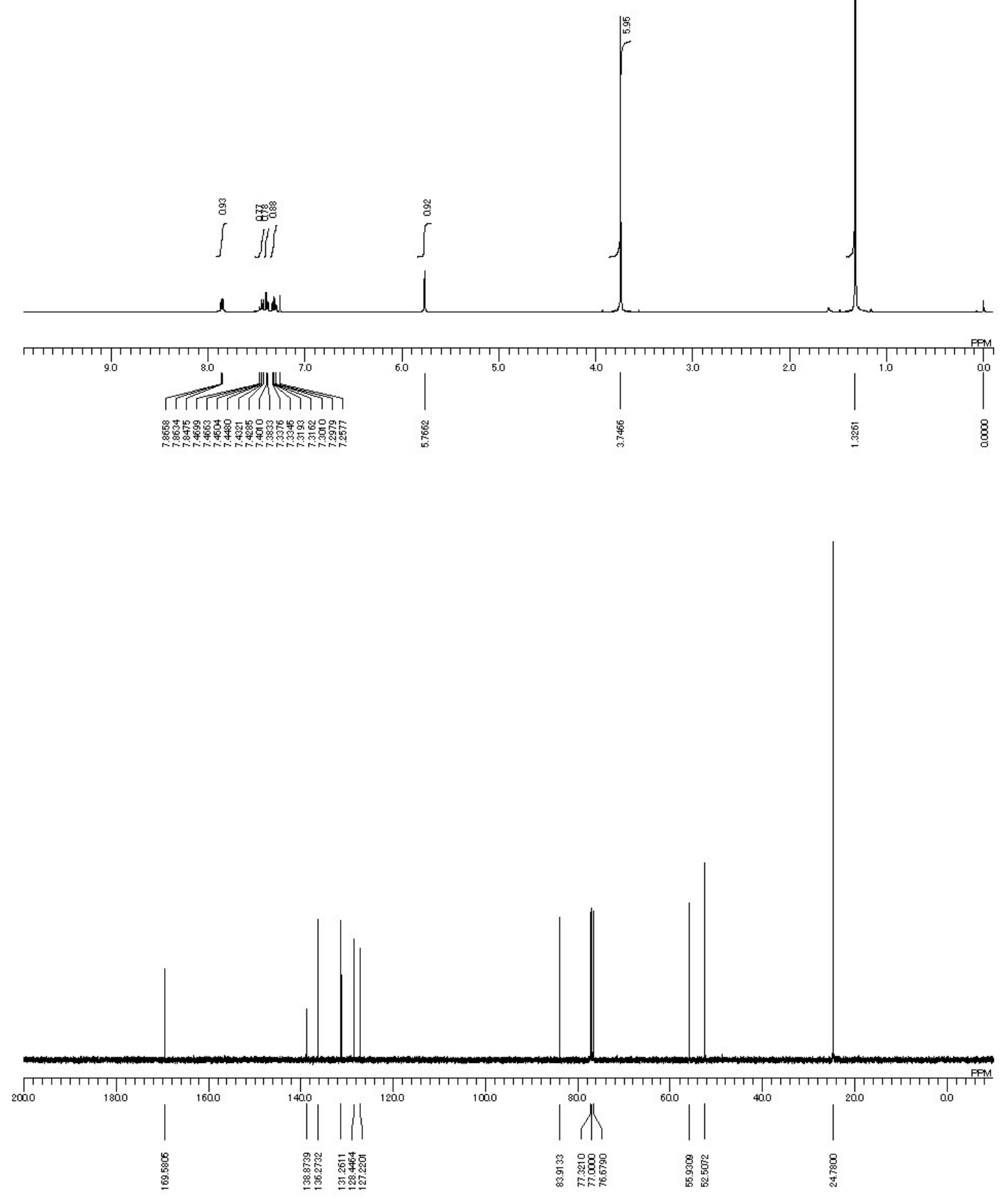

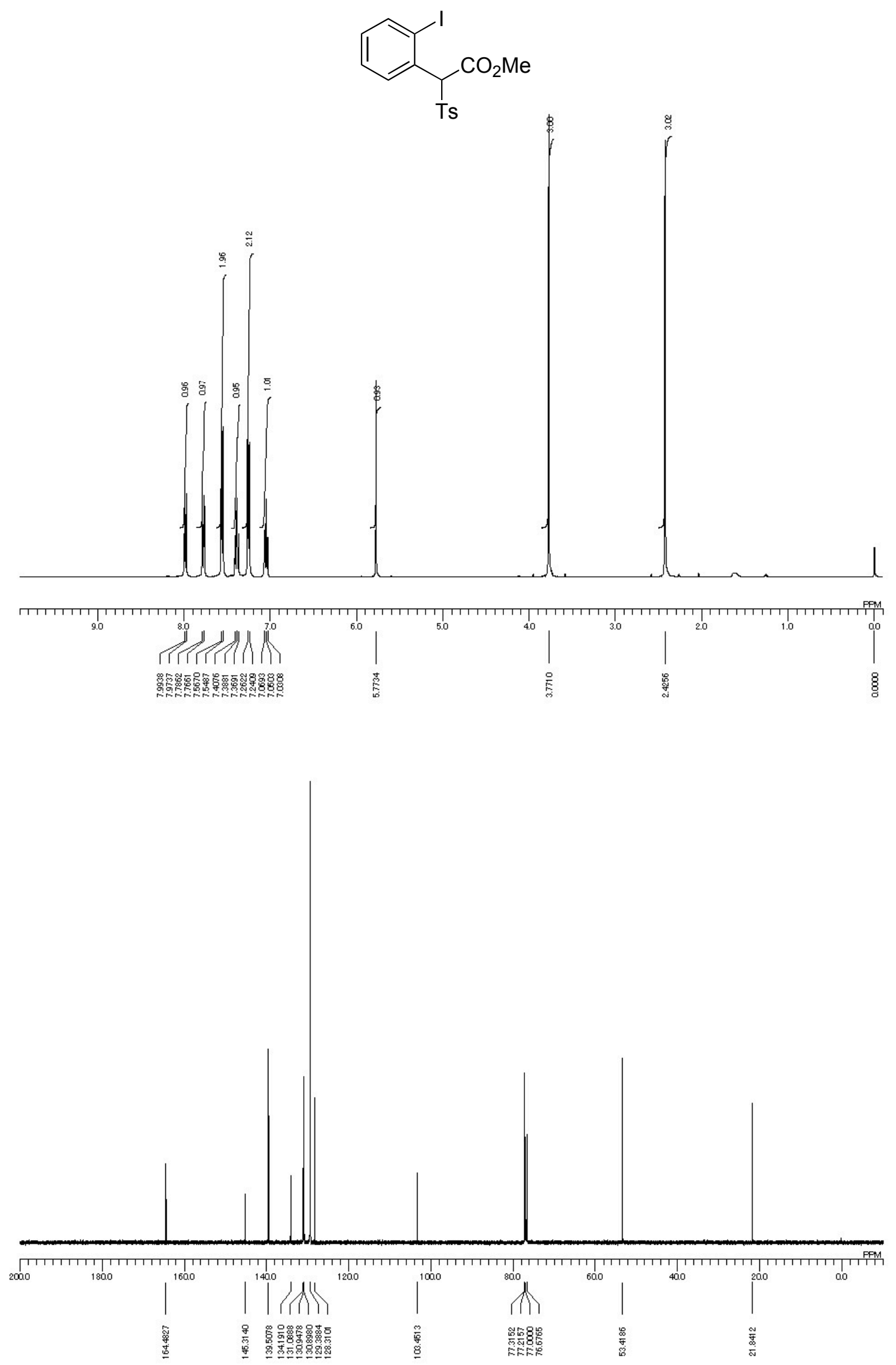


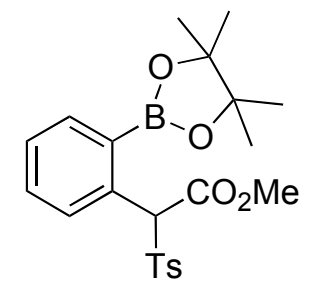

$14 c$
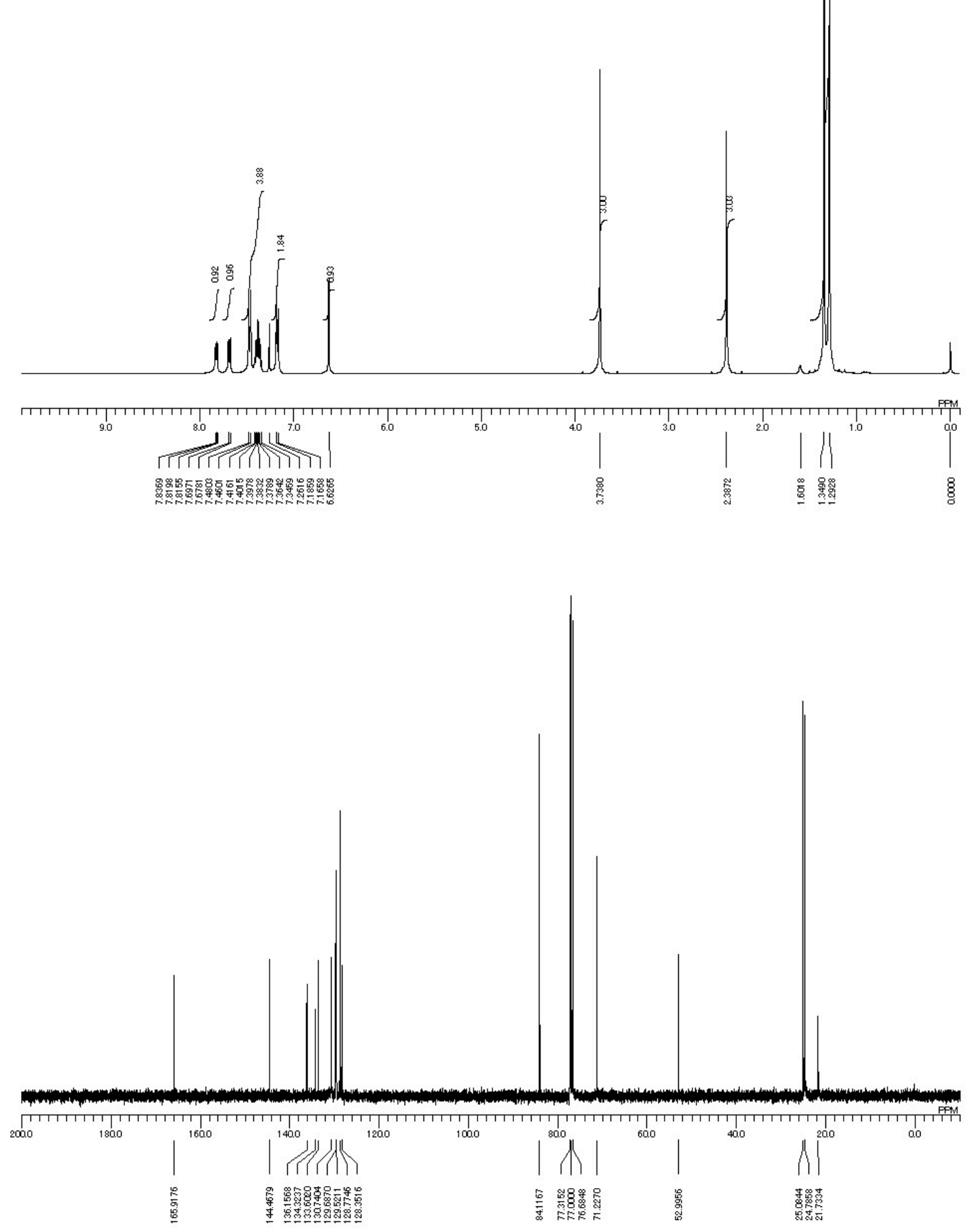

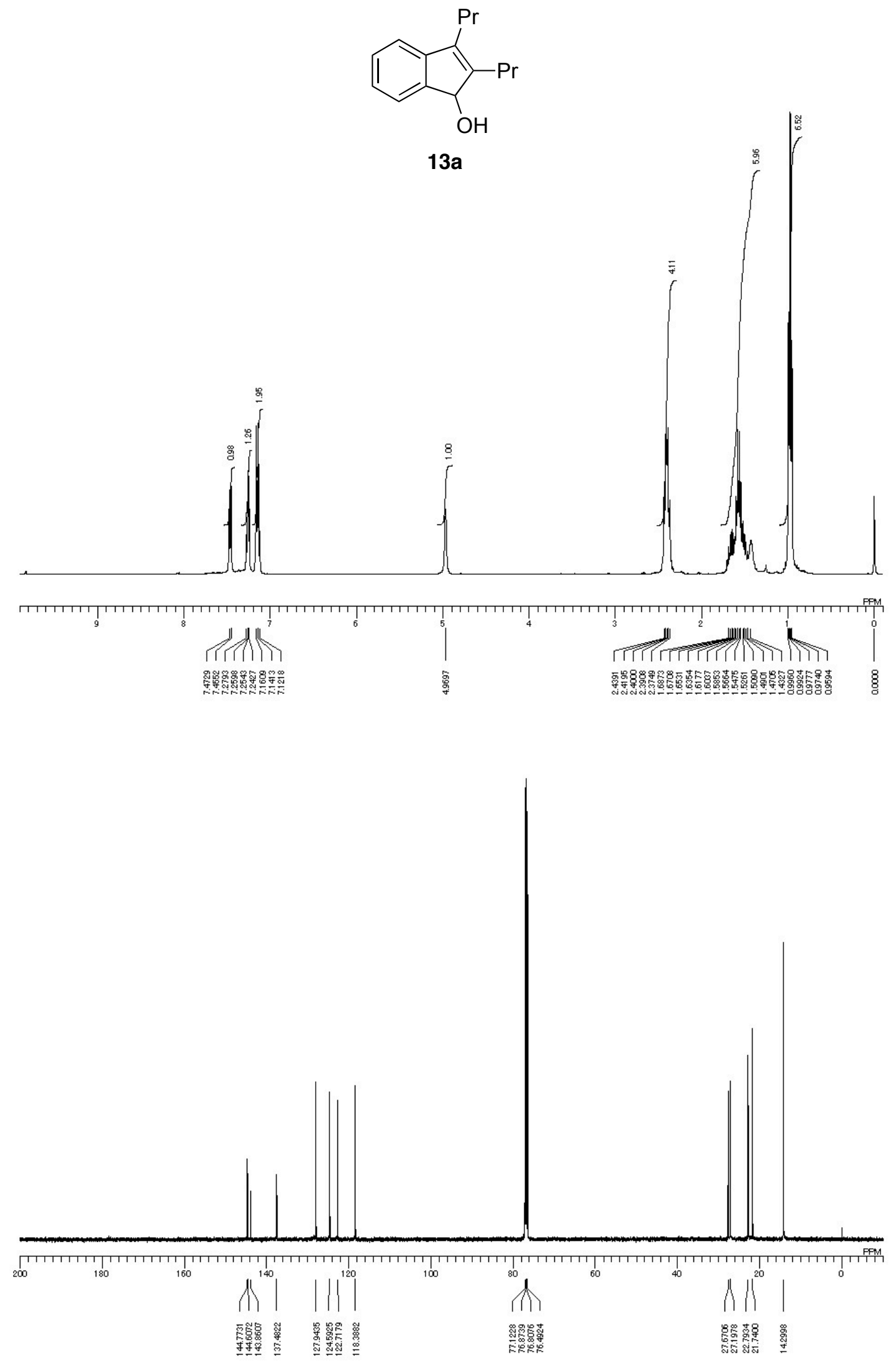


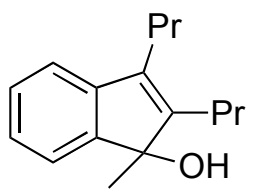

$13 b$
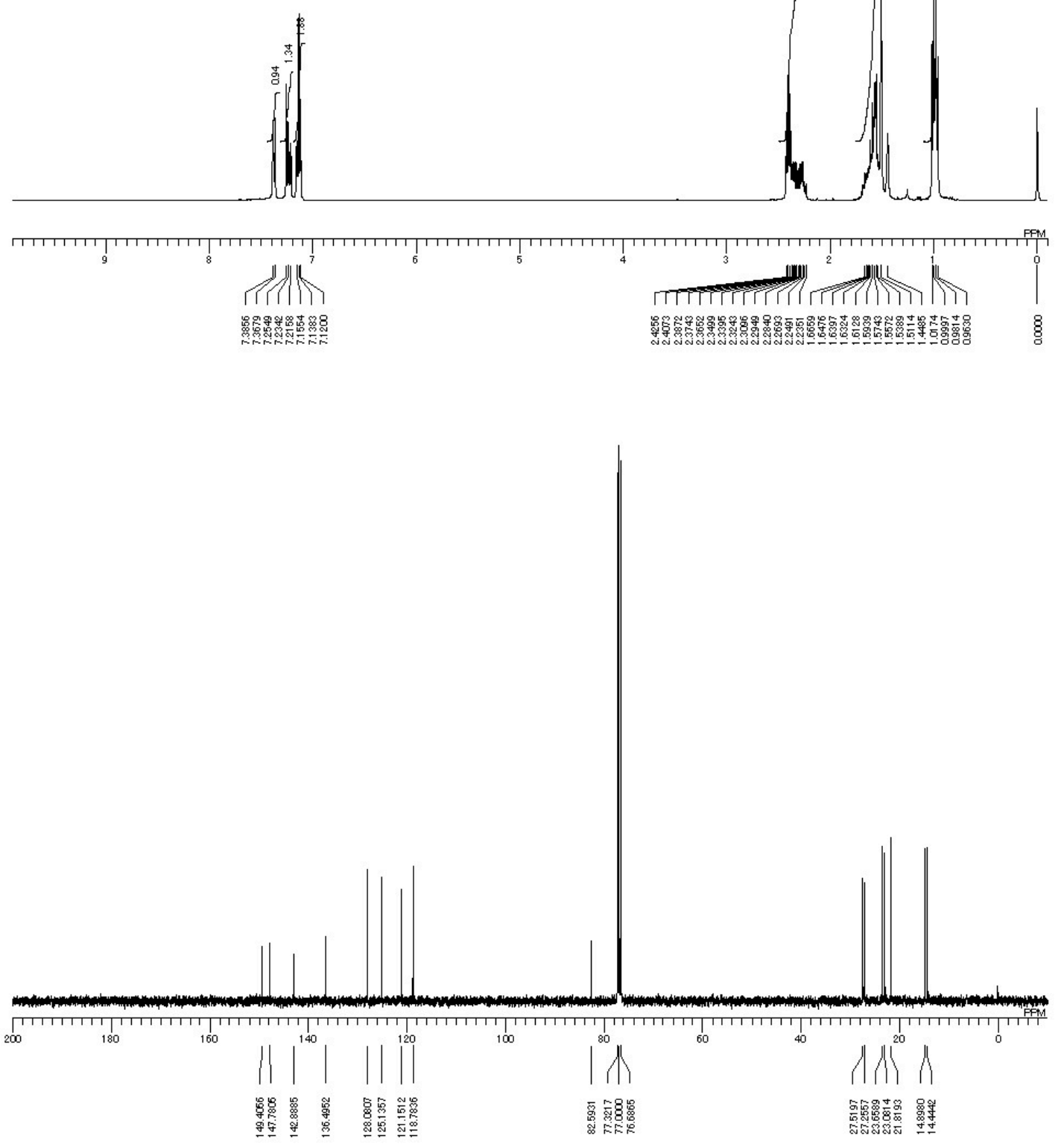

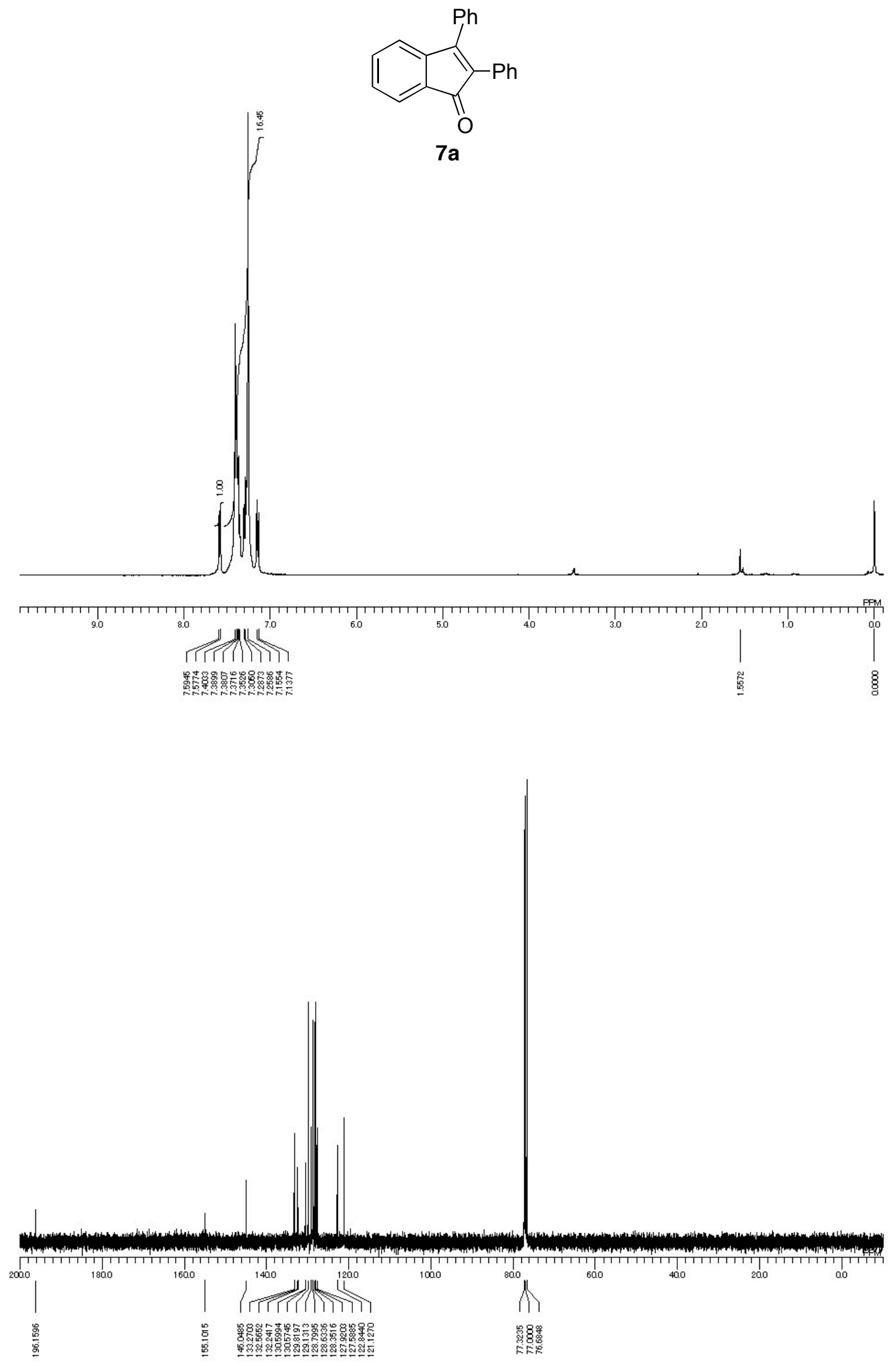
<smiles>COc1ccc(C2=C(c3ccc(OC)cc3)c3ccccc3C2=O)cc1</smiles>
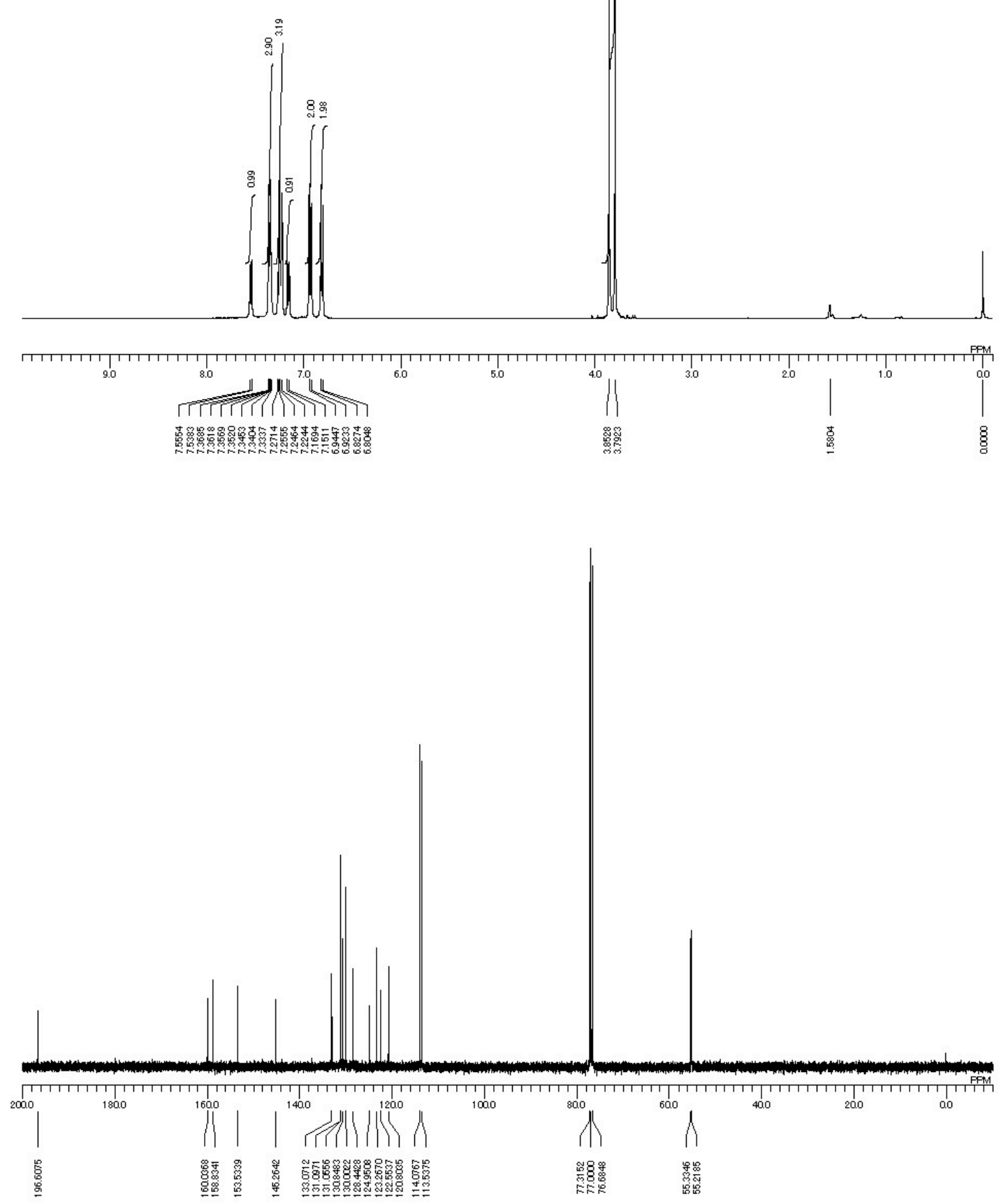

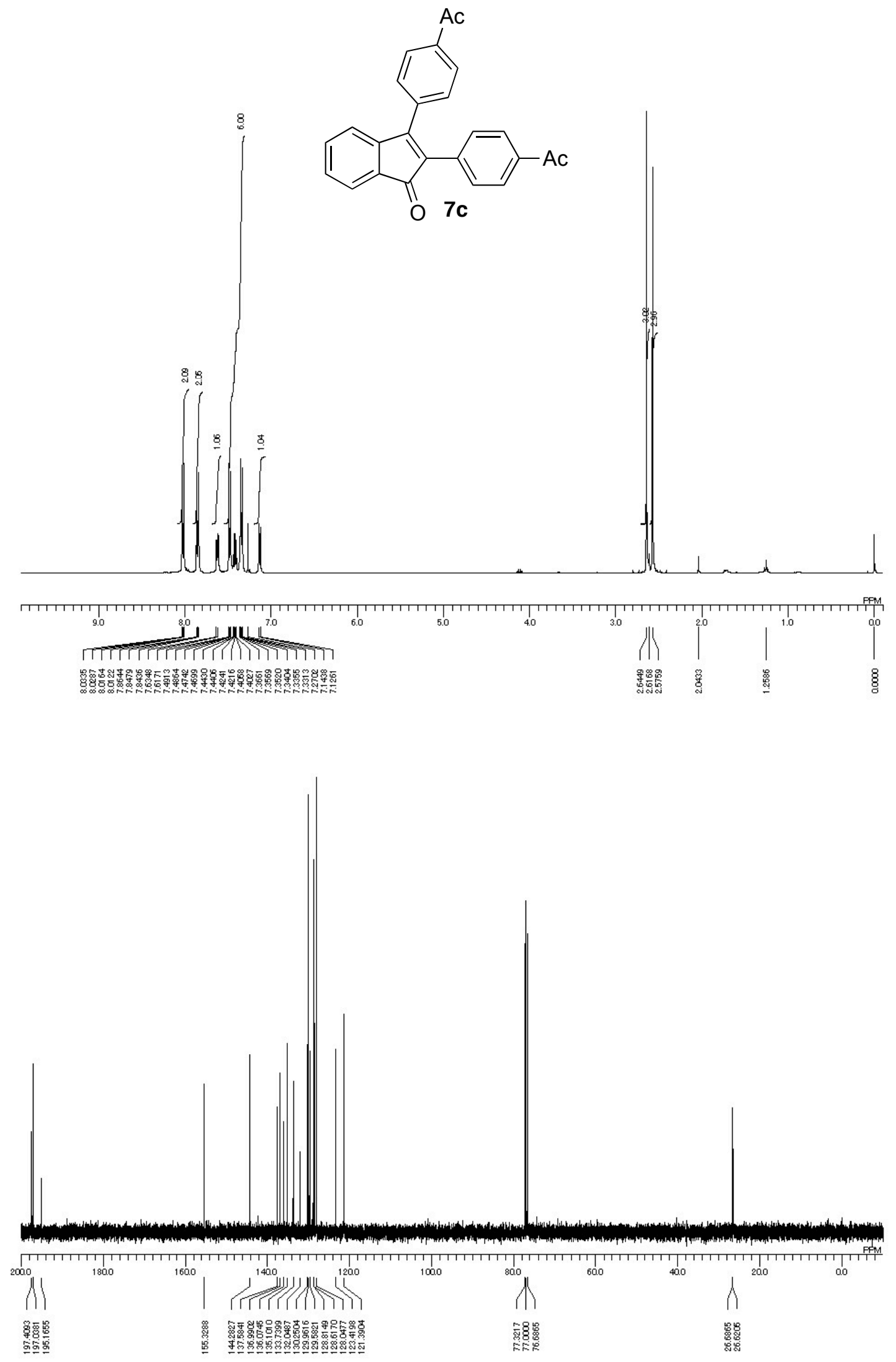

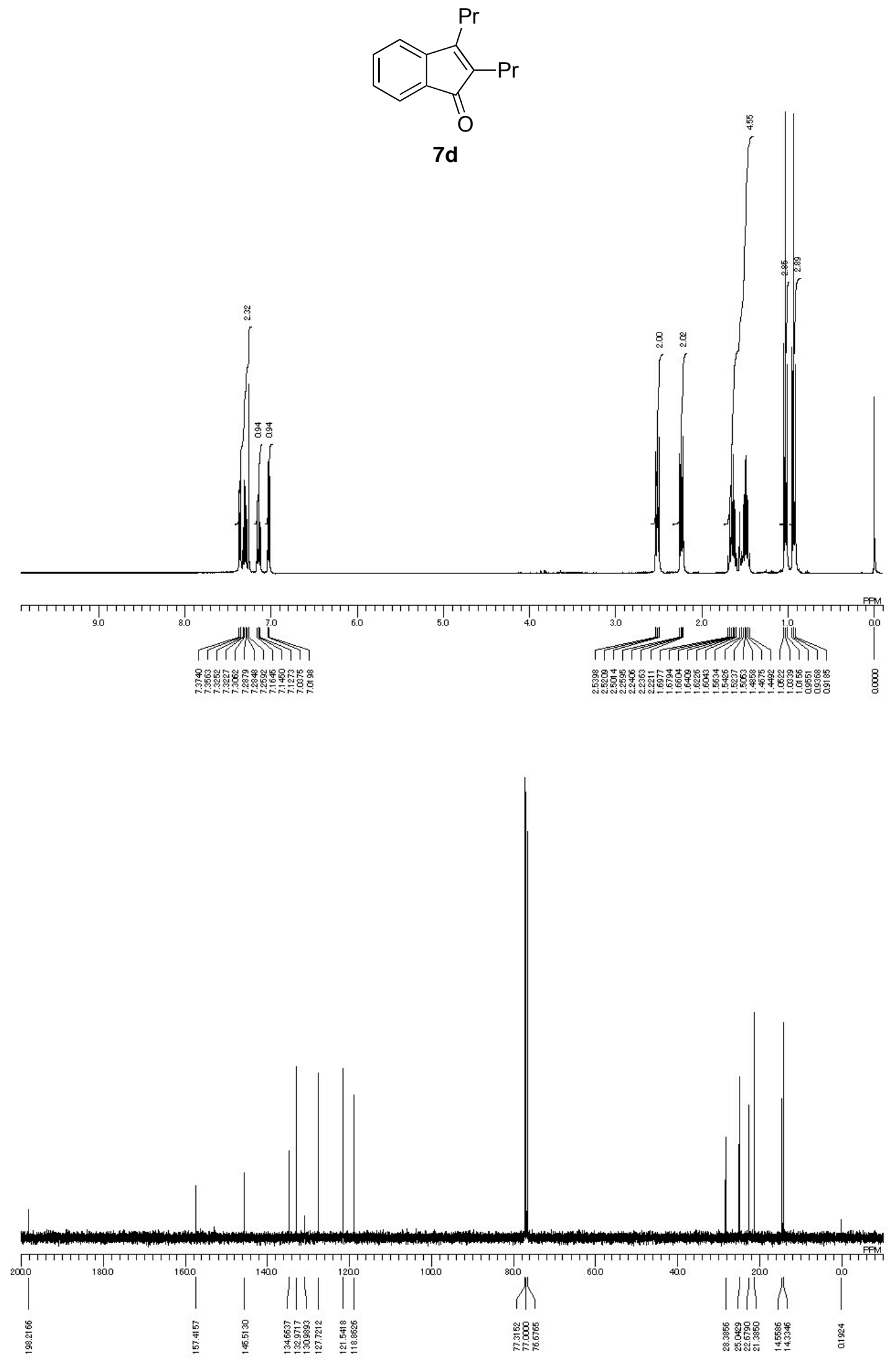

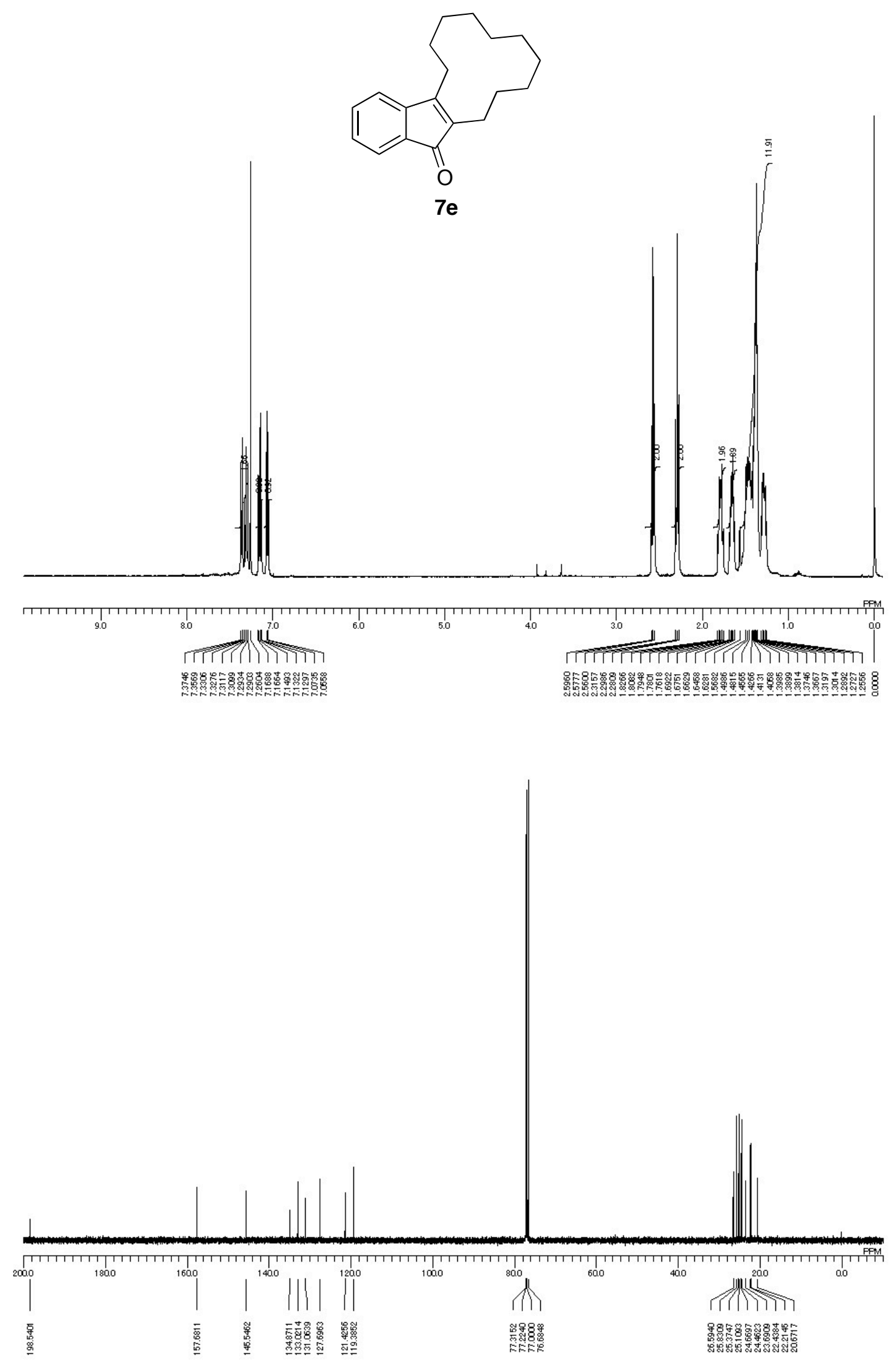

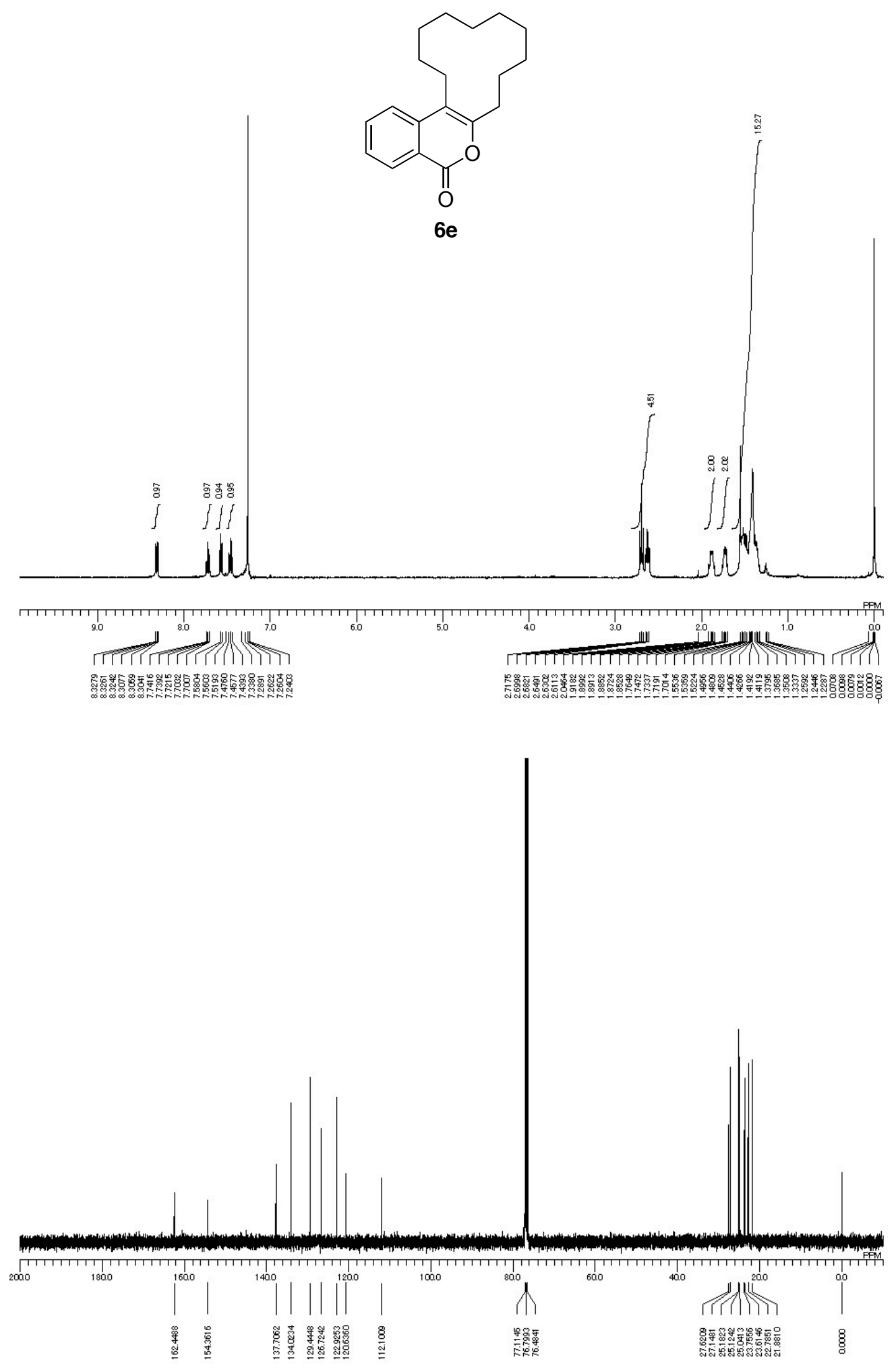

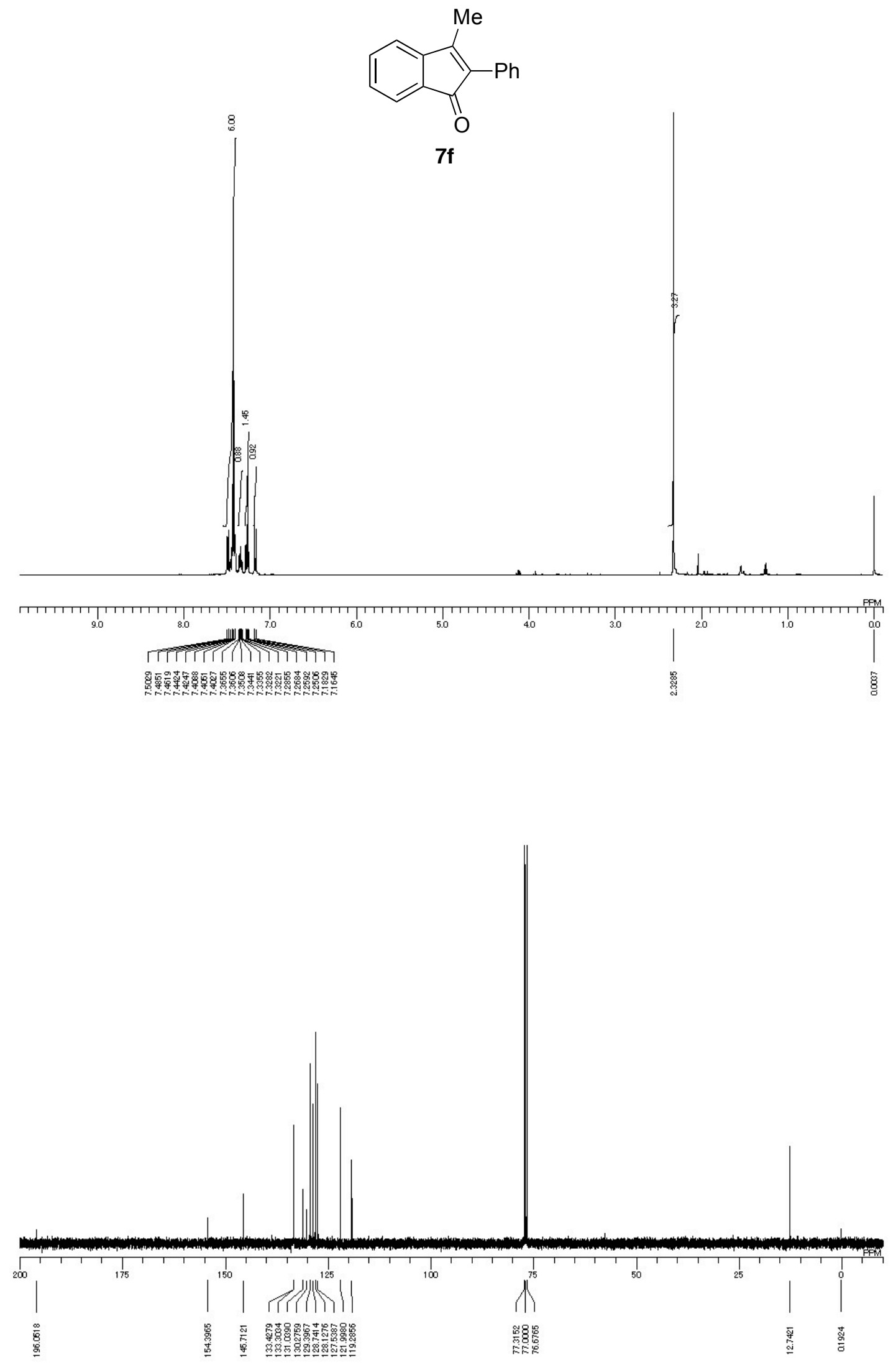

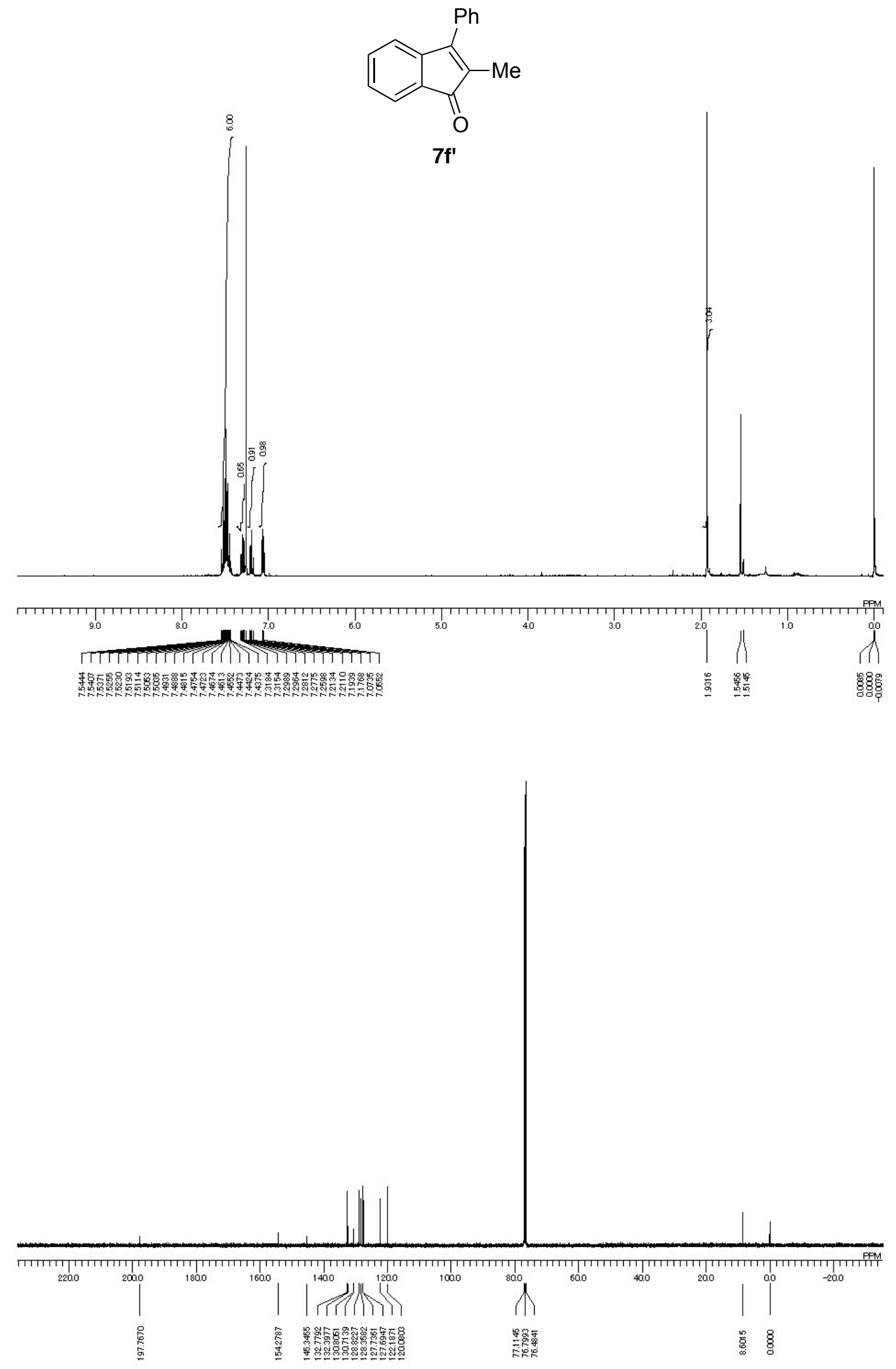

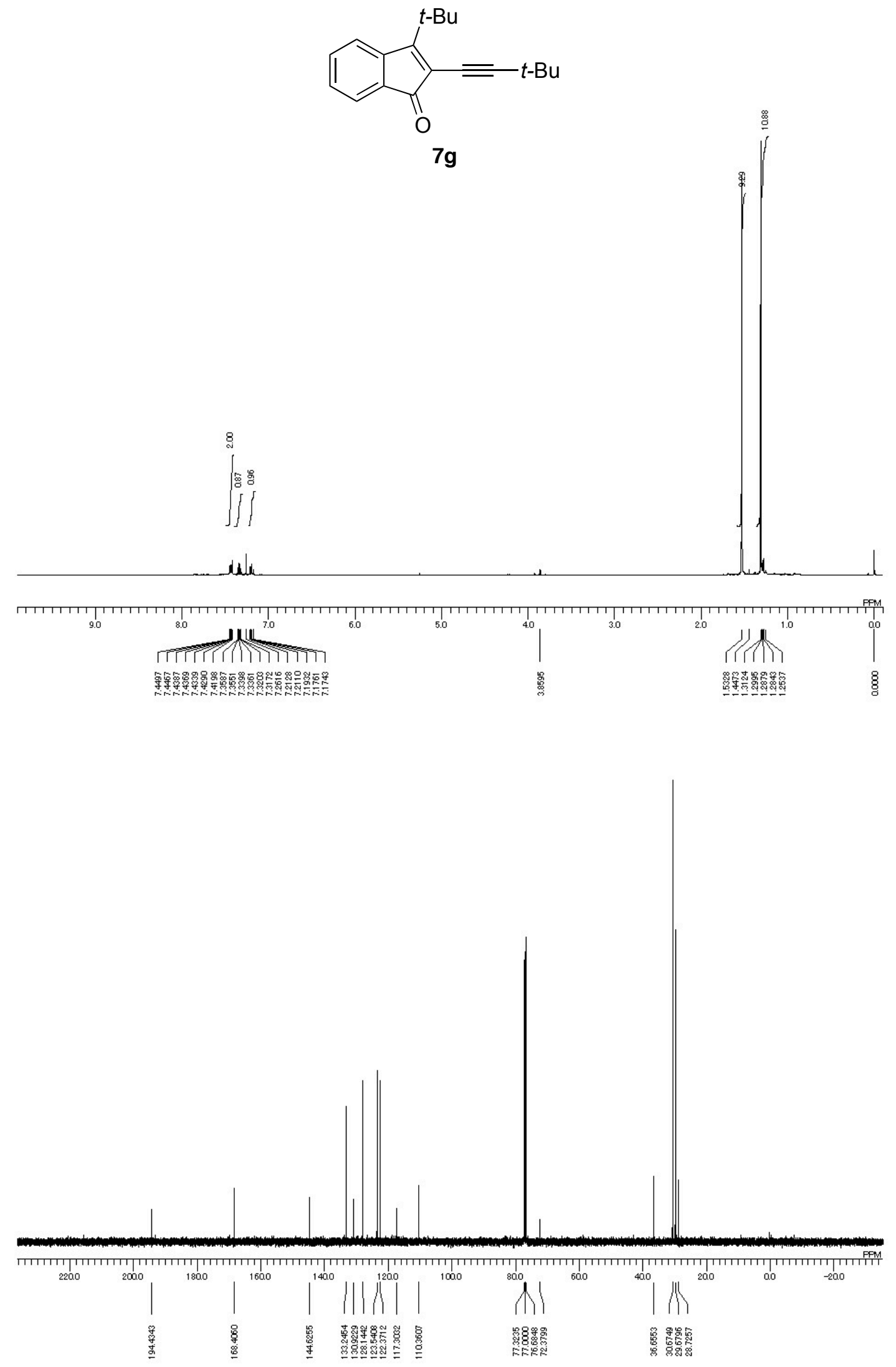

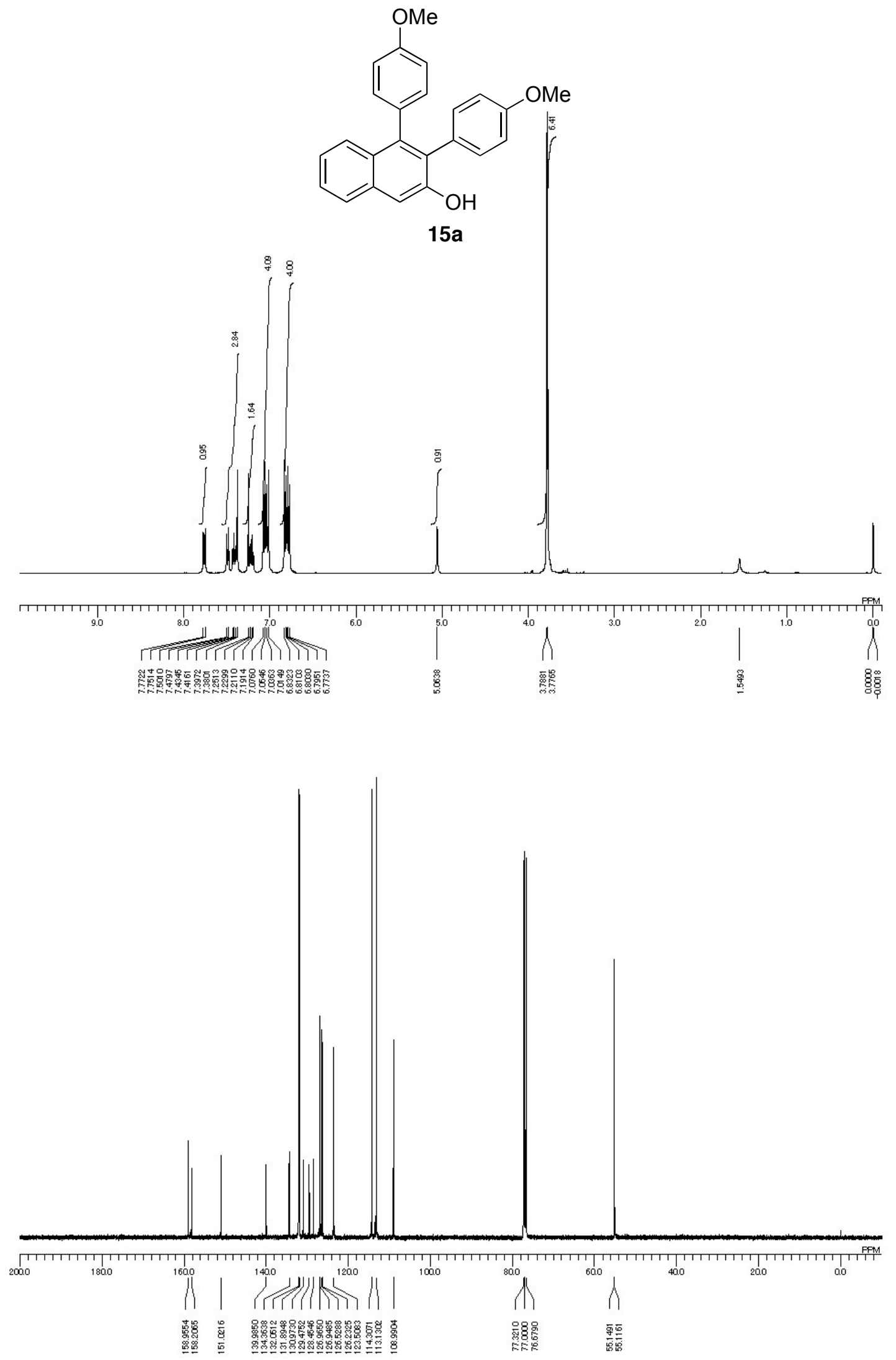

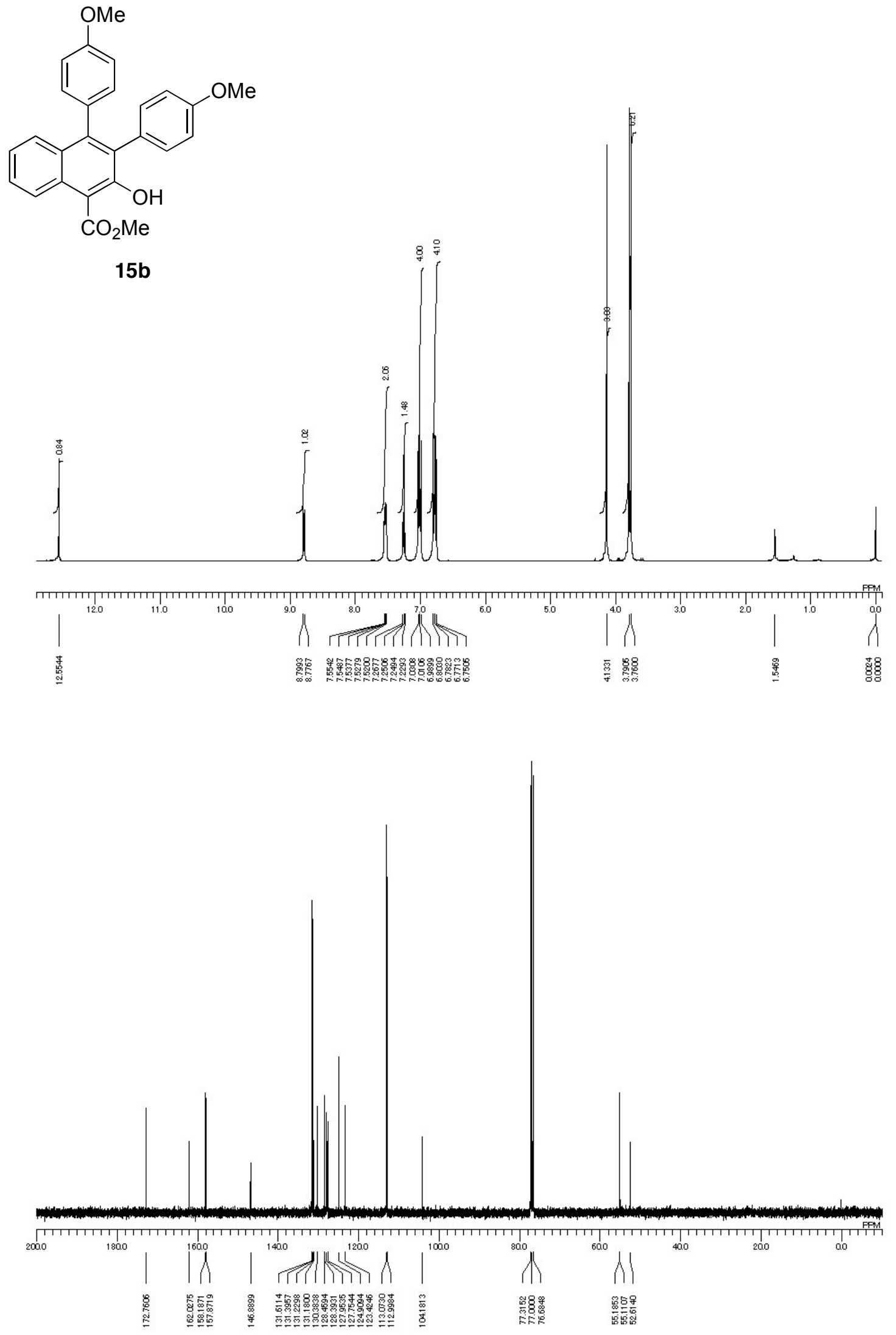

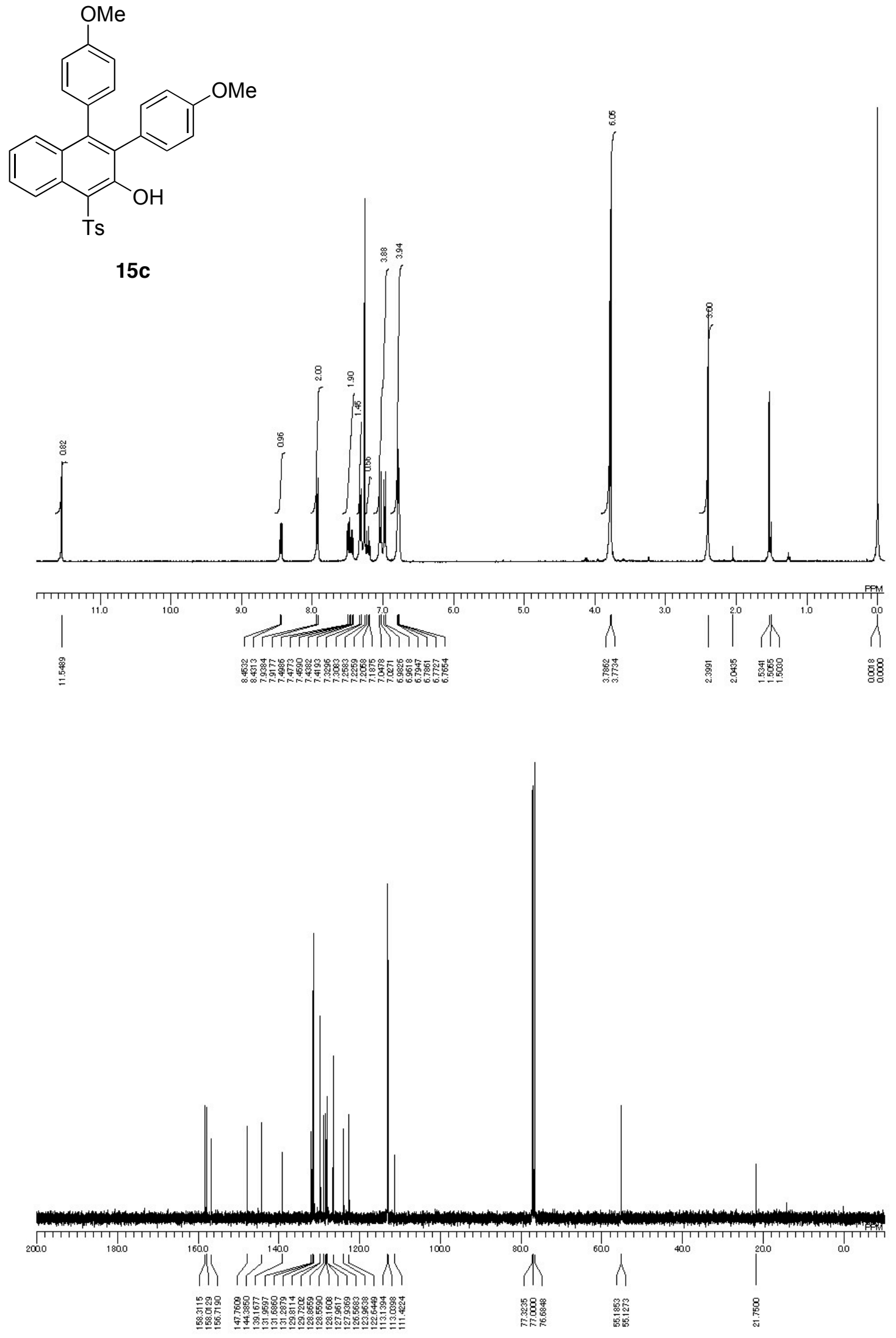